Article

\title{
Association of Finite-Dimension Thermodynamics and a Bond-Graph Approach for Modeling an Irreversible Heat Engine
}

\author{
Yuxiang Dong ${ }^{1,2}, *$, Amin El-Bakkali ${ }^{1}$, Michel Feidt ${ }^{3}$, Georges Descombes ${ }^{2}$ \\ and Christelle Périlhon ${ }^{2}$
}

1 Renault, Advanced Electronics and Technologies Division, Technocentre Renault, 1 avenue de Golf Guyancourt 78288, France; E-Mail: amin.el-bakkali@ renault.com

2 Laboratoire du Génie des Procédés pour l'Environnement, l'Énergie et la Santé (LGP2ES-EA21), Cnam-Cemagref, case 2D3R20, 292 rue saint Martin Paris 75003, France;

E-Mails: georges.descombes@ cnam.fr (G.D.); christelle.perilhon@ cnam.fr (C.P.)

3 Laboratoire d'Energétique et de Mécanique Théorique et Appliquée, ENSEM, 2, avenue de la Forêt de Haye Vandoeuvre 54516, France; E-Mail: michel.feidt@ensem.inpl-nancy.fr

* Author to whom correspondence should be addressed; E-Mail: yuxiang.dong@ renault.com.

Received: 23 April 2012; in revised form: 22 June 2012 / Accepted: 2 July 2012 /

Published: 12 July 2012

\begin{abstract}
In recent decades, the approach known as Finite-Dimension Thermodynamics has provided a fruitful theoretical framework for the optimization of heat engines operating between a heat source (at temperature $T_{h s}$ ) and a heat sink (at temperature $T_{c s}$ ). We will show in this paper that the approach detailed in a previous paper [1] can be used to analytically model irreversible heat engines (with an additional assumption on the linearity of the heat transfer laws). By defining two dimensionless parameters, the intensity of internal dissipation and heat leakage within a heat engine were quantified. We then established the analogy between an endoreversible heat engine and an irreversible heat engine by using the apparent temperatures $\left(T_{c s} \rightarrow T_{c s}^{\lambda, \varphi}, T_{h s} \rightarrow T_{h s}^{\lambda, \varphi}\right)$ and apparent conductances $\left(K_{h} \rightarrow K_{h}^{\lambda}, K_{c} \rightarrow K_{c}^{\lambda}\right)$. We thus found the analytical expression of the maximum power of an irreversible heat engine. However, these apparent temperatures should not be used to calculate the conversion efficiency at the optimal operating point by analogy with the case of an endoreversible heat engine.
\end{abstract}


Keywords: finite-dimension thermodynamics; bond graph approach; exo-reversible heat engine; irreversible heat engine; maximum power; Chambadal-Novikov-Curzon-Ahlborn efficiency

\section{Nomenclature}

\begin{tabular}{|c|c|c|}
\hline Variable & Unit & Description \\
\hline$T_{h s}$ & $\mathrm{~K}$ & Temperature of heat source \\
\hline$T_{c s}$ & $\mathrm{~K}$ & Temperature of heat sink \\
\hline$T_{h}$ & $\mathrm{~K}$ & Hot side temperature of heat engine \\
\hline$T_{c}$ & $\mathrm{~K}$ & Cold side temperature of heat engine \\
\hline$T_{s c}$ & $\mathrm{~K}$ & Cutoff temperature of heat engine \\
\hline$\dot{S}_{h s}$ & $\mathrm{~W} / \mathrm{K}$ & Entropy flow rate transferred at heat source \\
\hline$\dot{S}_{c s}$ & $\mathrm{~W} / \mathrm{K}$ & Entropy flow rate transferred at heat sink \\
\hline$\dot{S}$ & $\mathrm{~W} / \mathrm{K}$ & Entropy flow rate involved in reversible energy conversion \\
\hline$\dot{S}_{s c}$ & $\mathrm{~W} / \mathrm{K}$ & Cutoff entropy flow rate \\
\hline$\dot{S}_{o}$ & $\mathrm{~W} / \mathrm{K}$ & Optimal entropy flow rate \\
\hline$K_{\lambda}$ & $\mathrm{W} / \mathrm{K}$ & Internal thermal conductance of heat engine \\
\hline$K_{l}$ & $\mathrm{~W} / \mathrm{K}$ & Conductance of heat leakage between heat source and heat sink \\
\hline$K_{h}$ & $\mathrm{~W} / \mathrm{K}$ & Global thermal conductance of heat exchanger at hot side \\
\hline$K_{c}$ & $\mathrm{~W} / \mathrm{K}$ & Global thermal conductance of heat exchanger at cold side \\
\hline$K_{s c}$ & $\mathrm{~W} / \mathrm{K}$ & Equivalent thermal conductance \\
\hline$\dot{\sigma}^{e x}$ & $\mathrm{~W} / \mathrm{K}$ & Rate of total entropy generation within exo-reversible heat engine \\
\hline$\dot{\sigma}_{\lambda}^{e x}$ & $\mathrm{~W} / \mathrm{K}$ & Rate of entropy generation related to internal heat transfer \\
\hline$\dot{\sigma}_{\varphi}^{e x}$ & $\mathrm{~W} / \mathrm{K}$ & Rate of entropy generation related to internal dissipation \\
\hline$\dot{Q}_{h s}$ & W & Thermal power supplied by heat source \\
\hline$\dot{Q}_{c s}$ & $\mathrm{~W}$ & Thermal power received by heat link \\
\hline$\dot{Q}_{h}$ & $\mathrm{~W}$ & Thermal power exchanged between heat source and heat engine \\
\hline$\dot{Q}_{c}$ & $\mathrm{~W}$ & Thermal power exchanged between heat sink and heat engine \\
\hline$\dot{Q}_{\lambda}$ & $\mathrm{W}$ & Internal heat leakage \\
\hline$\dot{Q}_{l}$ & $\mathrm{~W}$ & External heat leakage between heat source and heat link \\
\hline$\dot{Q}_{s c}$ & $\mathrm{~W}$ & Cutoff thermal power \\
\hline$\Phi$ & $\mathrm{W}$ & Internal dissipation within heat engine \\
\hline$\dot{q}_{h}$ & $\mathrm{~W}$ & Input thermal power of converter \\
\hline$\dot{q}_{c}$ & $\mathrm{~W}$ & Output thermal power of converter \\
\hline$\dot{W}$ & $\mathrm{~W}$ & Output mechanical power of heat engine \\
\hline$\dot{W}_{o}$ & $\mathrm{~W}$ & Maximum mechanical power of heat engine \\
\hline$\eta_{1}$ & -- & Energy conversion efficiency \\
\hline$\eta_{o}$ & -- & Energy conversion efficiency at optimal operating point of heat engine \\
\hline$\eta_{C}$ & -- & Carnot efficiency \\
\hline
\end{tabular}




$\begin{array}{lll}\text { Variable } & \text { Unit } & \begin{array}{l}\text { Description } \\ \text { Chambadal-Novikov-Curzon-Ahlborn efficiency }\end{array} \\ \eta_{C N C A} & -- & \text { Figure of merit of thermoelectric material at temperature } \mathrm{T} \\ Z T & -- & \begin{array}{l}\text { dimensionless number of internal dissipation within heat engine } \\ \text { dimensionless number of internal heat loss within heat engine }\end{array} \\ \varphi & -- & \text { Distribution parameter of internal dissipation at hot side } \\ \lambda & -- & \text { Distribution parameter of internal dissipation at cold side } \\ f_{h} & -- & \text { Internal "resistance" of heat engine } \\ f_{c} & -- & \text { External "resistance" of heat engine } \\ F^{\varphi} & K^{2} / W & \text { Internal electric resistance of thermoelectric generator } \\ F_{\text {load }}^{\varphi} & K^{2} / W & \text { Heat exchange surface } \\ R & \Omega & \text { Heat exchange coefficient } \\ A & m^{2} & \text { Electric current of thermoelectric generator } \\ U & W / K / m^{2} & \text { Coefficient of Seebeck } \\ I & \mathrm{~A} & \text { Open circuit voltage of thermoelectric generator } \\ \alpha & \mathrm{V} / \mathrm{K} & \mathrm{V}\end{array}$

\section{Introduction}

In recent decades, the approach known as Finite-Dimension Thermodynamics has provided a fruitful theoretical framework for the optimization of heat engines operating between a heat source (at temperature $T_{h s}$ ) and a heat sink (at temperature $T_{c s}$ ) [2-15]. The main idea of this approach is the coupling of a converter with two heat exchangers of finite dimension which connect the converter to thermostats. In other words, in the framework of Finite-Dimension Thermodynamics, we take into account at least the external irreversibilities (Figure 1). This approach was initiated independently by Chambadal [16] and Novikov [17] in 1957 and then clarified by Feidt [18] and others [19-23]. The simple case where only the external irreversibilities related to external heat transfer between thermostats and converter are taken into account corresponds to the case that we call an "Endoreversible Heat Engine". The most remarkable result of this theoretical case (with an additional assumption on the linearity of the laws of heat transfer) is the Chambadal-Novikov-Curzon-Ahlborn efficiency $\eta_{C N C A}=1-\sqrt{ } T_{C s} / T_{h s}$ aobtained at the optimal operating point where the output mechanical power is maximized. This efficiency, like that of the ideal Carnot engine $\eta_{C}=1-T_{c s} / T_{h s}$, depends only on the temperatures of heat source and heat sink.

There has been much discussion about the energy conversion efficiency at the optimal operating point of an endoreversible heat engine and an exo-reversible heat engine [24-35]. In particular, Apertet et al. [28] developed an empirical model of a thermoelectric generator and then compared the impact of internal and external irreversibilities on the conversion efficiency at the optimal point where the electrical power is maximized. They showed that the Chambadal-Novikov-Curzon-Ahlborn efficiency $\eta_{C N C A}$ is only available for endoreversible heat engines, whereas the Schmiedl-Seifert efficiency $\eta_{S S}$ [27] applies only to exo-reversible heat engines where the irreversibilities are fully internal. In another paper, Apertet et al. [29] studied the conversion efficiency at maximum power of a system composed of two thermoelectric generators thermally connected in series but electrically independent. By neglecting the heat loss within the thermoelectric generators, they showed that the optimum 
condition for maximum power is not unique but depends on the combination of the electrical load resistances of each generator.

Figure 1. Heat engine classification according to internal and external irreversibilities.

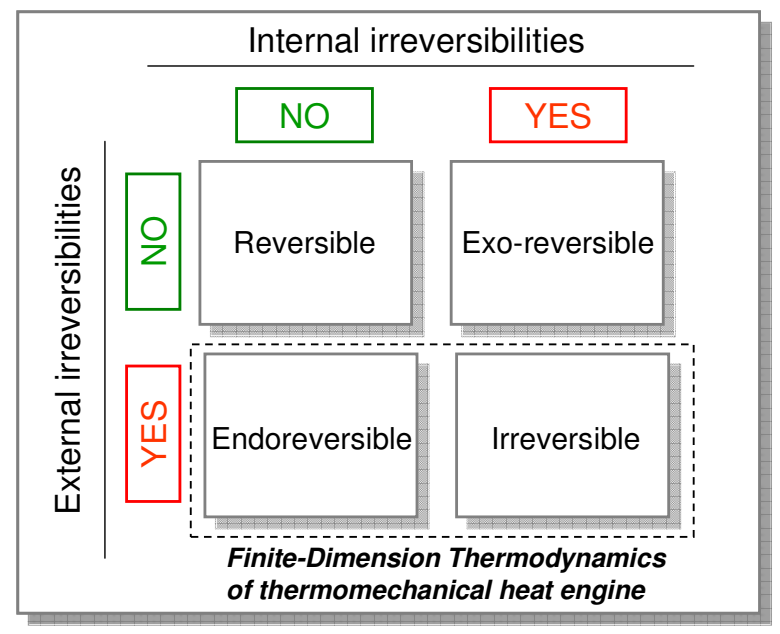

In this paper, we will firstly recall the notion of an Exo-reversible Heat Engine where only the internal irreversibilities are taken into account [36,37], and then by applying a new approach [1] based on the association of Finite-Dimension Thermodynamics and the Bond-Graph approach [38,39], we give the analytical expressions of the optimal operating point of an Irreversible Heat Engine where the energy conversion is accompanied by irreversibilities related to internal heat leakage and internal dissipation. An application of this approach to a thermoelectric generator [40,41] allows one to optimize the design of the machine and express the energy recovery potential based on the physical parameters of the system.

We chose the optimization criterion based on the maximum mechanical power $[11,42,43]$ as it is a relevant criterion for heat recovery systems in which the heat source is considered "free" such as the exhaust gas of a motor vehicle. These heat recovery systems (ORC system [44,45], thermoelectric generator $[40,41]$ ) are potentially interesting in view of the technical solutions designed to reduce the TCO (Total Cost of Ownership) of vehicles and greenhouse gas emissions.

\section{Modeling of Exo-Reversible Heat Engine at Steady State}

Figure 2 shows the Bond-Graph diagram of an exo-reversible heat engine where only the internal irreversibilities are taken into account. We consider two sources of irreversibilities here: internal dissipation and heat leakage.

In the case of exo-reversible heat engine, we assume that there is no heat leakage between thermostats and that the thermal conductances between the heat engine and the thermostats are infinite. As a result, there is no thermal gradient between the machine and the thermostats $\left(T_{h}=T_{h s}, T_{c}=T_{c s}\right)$, which means that the entropy generation in these conductances is zero. In fact, the rate of entropy generation by heat transfer is given by: $\dot{\sigma}=\dot{Q} \cdot \Delta\left(\frac{1}{T}\right)=-\frac{\dot{Q}^{2}}{K T^{2}}$. Since the thermal power transferred $\dot{Q}$ is finite and the thermal conductance $K$ is infinite, the rate of entropy generation is zero. 
Figure 2. Bond-Graph diagram of an exo-reversible heat engine at steady state.

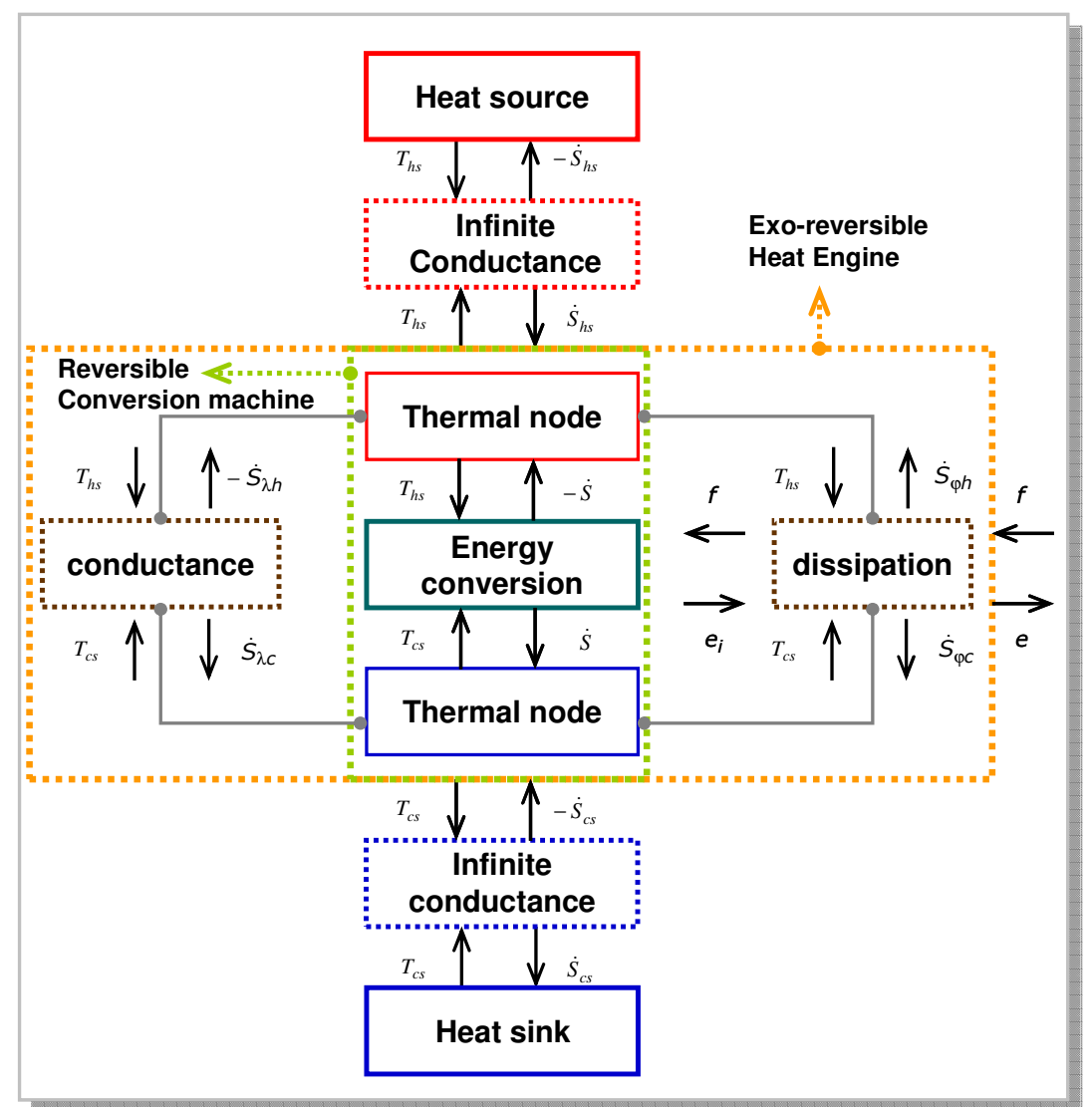

By convention, the arrows next to the flow variables indicate the positive direction of power transfer. For example, the entropy flow rate from the heat source to the exo-reversible heat engine is the term $\dot{S}_{h s}$ while the entropy flow rate from the exo-reversible heat engine to the heat sink is the term $\dot{S}_{c s}$ (Figure 2). To remain consistent with the case of the endoreversible heat engine that we detailed in another article [1], we call $\dot{S}$ the entropy flow rate involved in reversible energy conversion and we use it as control variable of the exo-reversible heat engine. Similarly, we keep the same notation for the energy flow rates at the border of the machine: $\dot{Q}_{h}, \dot{Q}_{c}$ for the thermal powers at heat source and heat sink and $\dot{W}=\dot{Q}_{h}-\dot{Q}_{c}$ for the output mechanical power (Figure 3).

To simplify the problem in order to obtain analytical solutions, we make the following assumptions:

- The temperatures of heat source and heat sink are constant $\left(T_{h s}\right.$ and $\left.T_{c s}\right)$.

- The energy conversion is a reversible process (conservation of entropy flow rate $\left.\dot{S} \Leftrightarrow \dot{q}_{h}=\dot{S} T_{h s}, \dot{q}_{c}=\dot{S} T_{c s}\right)$.

- The law of heat transfer is linear (constant conductance $K_{\lambda}$ ).

- The internal dissipation $\Phi$ depends only on the control variable $\dot{S}: \Phi=\Phi(\dot{S})$. This assumption can be justified when the entropy flow rate $\dot{S}$ involved in reversible energy conversion completely defines the operating point of the machine. Moreover, in the case of a thermoelectric generator where the internal dissipation is related to the Joule effect, we have: $\Phi=R \cdot I^{2}, I=-\frac{\dot{S}}{\alpha} \Rightarrow \Phi=F^{\varphi} \dot{S}^{2}, F^{\varphi}=\frac{R}{\alpha^{2}}$ where $R$ is the electric resistance of the thermoelectric generator and $\alpha$ the Seebeck coefficient. 
Figure 3. Power balance of an exo-reversible heat engine.

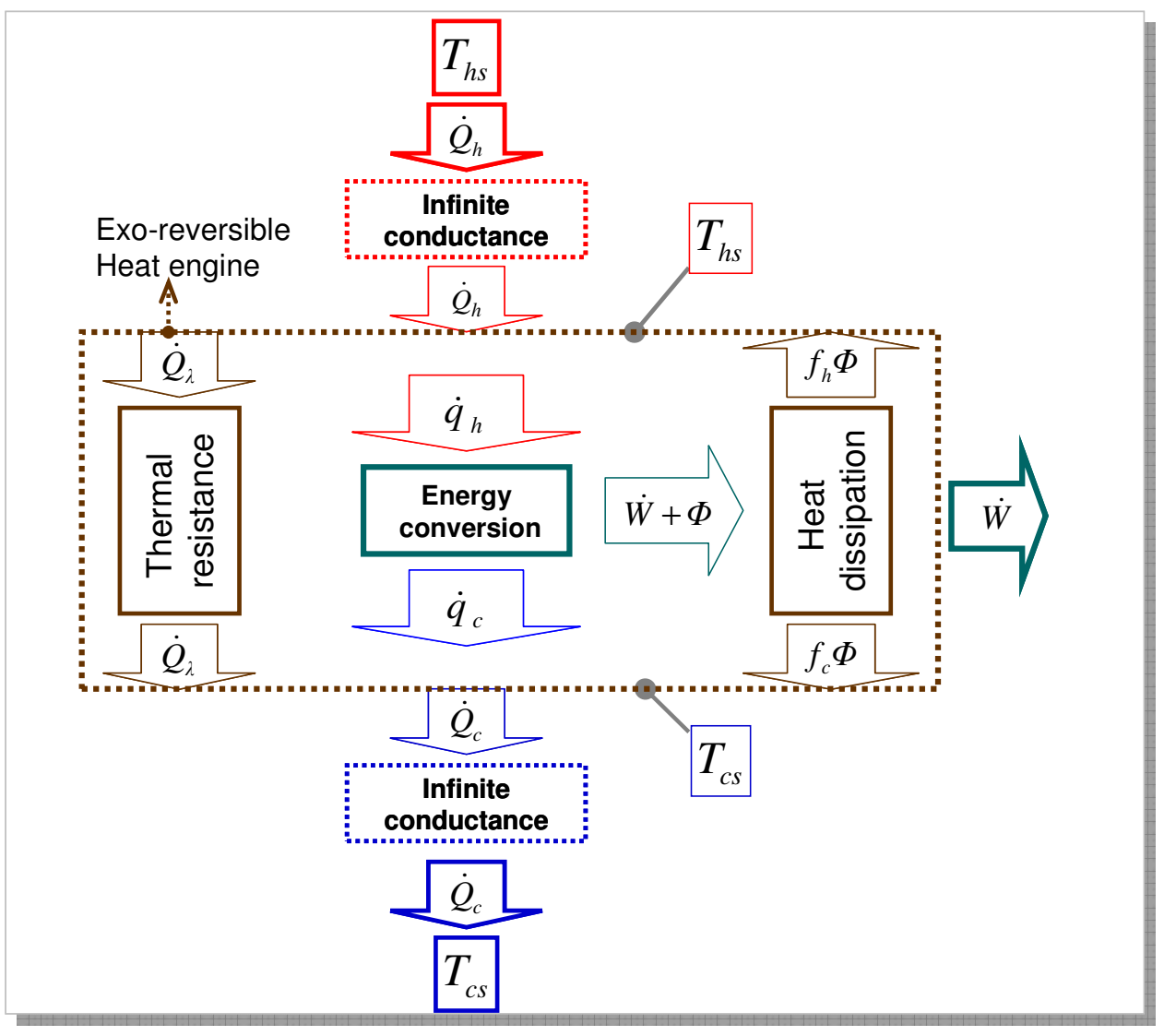

\subsection{Energy Balance at Steady State}

With the assumptions of steady state and linearity of the laws of heat transfer, we obtain the following equations ( $c f$. Figure 3 ):

$$
\begin{gathered}
\dot{Q}_{\lambda}=K_{\lambda}\left(T_{h s}-T_{c s}\right) \\
\dot{Q}_{h}=\dot{q}_{h}+\dot{Q}_{\lambda}-f_{h} \Phi \\
\dot{Q}_{c}=\dot{q}_{c}+\dot{Q}_{\lambda}+f_{c} \Phi \\
\dot{W}=\dot{Q}_{h}-\dot{Q}_{c}=\left(\dot{q}_{h}-\dot{q}_{c}\right)-\Phi
\end{gathered}
$$

where $f_{c}$ and $f_{h}$ are two coefficients which determine the distribution of the internal dissipated power $\Phi$ between the hot and cold sides $\left(f_{c}+f_{h}=1\right)$. To simplify our problem, we make an additional assumption that the internal dissipated power $\Phi$ is proportional to the square of the entropy flow rate $\dot{S}$ (Joule friction):

$$
\Phi=F^{\varphi} \dot{S}^{2}
$$

where $F^{\varphi}$ is the internal "resistance" assumed constant. We then obtain the expressions of the thermal powers $\dot{Q}_{h}, \dot{Q}_{c}$, the output mechanical power $\dot{W}$ and the energy conversion efficiency $\eta_{1}$ of the exo-reversible heat engine in terms of entropy flow rate $\dot{S}$ ( $c f$. Figure 2):

$$
\dot{Q}_{h}=K_{\lambda}\left(T_{h s}-T_{c s}\right)+\dot{S} T_{h s}-f_{h} F^{\varphi} \dot{S}^{2}
$$




$$
\begin{gathered}
\dot{Q}_{c}=K_{\lambda}\left(T_{h s}-T_{c s}\right)+\dot{S} T_{c s}+f_{c} F^{\varphi} \dot{S}^{2} \\
\dot{W}=\dot{S}\left(T_{h s}-T_{c s}\right)-F^{\varphi} \dot{S}^{2} \\
\eta_{1}=\frac{\dot{W}}{\dot{Q}_{h}}=\frac{\left(T_{h s}-T_{c s}\right) \dot{S}-F^{\varphi} \dot{S}^{2}}{K_{\lambda}\left(T_{h s}-T_{c s}\right)+\dot{S} T_{h s}-f_{h} F^{\varphi} \dot{S}^{2}}
\end{gathered}
$$

When we vary the entropy flow rate $\dot{S}$, the operating point of the machine moves. Relation (7) forms the parametric equations of the operating curve of the exo-reversible heat engine that we are going to study in the $\left[\dot{W}, \eta_{1}\right]$ diagram (cf. Figure 4).

Figure 4. Operating curve of an exo-reversible heat engine.
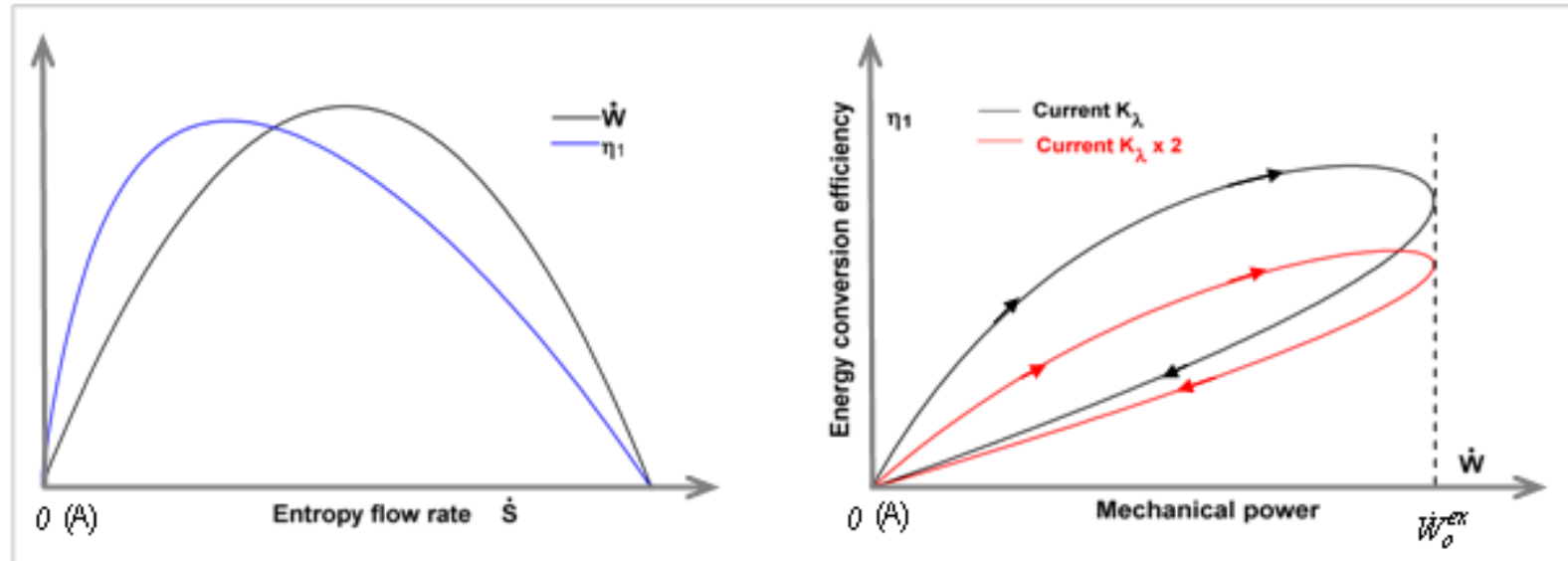

\subsection{Determination of the Maximum Mechanical Power and the Associated Efficiency}

The range of variation of the entropy flow rate $\dot{S}$ is implicitly defined by the inequality $\dot{W}(\dot{S}) \geq 0$. We immediately obtain the cutoff entropy flow rate $\dot{S}_{s c}^{\text {ex }}$, a nontrivial solution of $\dot{W}(\dot{S})=0$ :

$$
\dot{S}_{s c}^{\mathrm{ex}}=\frac{T_{h s}-T_{c s}}{F^{\phi}}
$$

By deriving the mechanical power given by Equation (7) with regard to the entropy flow rate involved in the energy conversion $\dot{S}$, we easily deduce the expression of the optimal entropy flow rate $\dot{S}_{o}^{e x}$ for which we obtain the maximum power of the machine:

$$
\frac{d \dot{W}}{d \dot{S}}=T_{h s}-T_{c s}-2 F^{\varphi} \dot{S} \rightarrow \dot{S}_{o}^{\mathrm{ex}}=\frac{T_{h s}-T_{c s}}{2 \cdot F^{\varphi}}=\frac{\dot{S}_{s c}^{\mathrm{ex}}}{2}
$$

we note that the optimal entropy flow rate $\dot{S}_{o}^{\text {ex }}$ is equal to half of the cutoff entropy flow rate $\dot{S}_{s c}^{\mathrm{ex}}$ in the case of an exo-reversible heat engine which recalls a well-known result in the field of thermoelectric conversion [46]. By injecting the optimal entropy flow rate in (7), we obtain the expression of maximum output power of the exo-reversible heat engine:

$$
\dot{W}_{o}^{\mathrm{ex}}=\frac{\left(T_{h s}-T_{c s}\right)^{2}}{4 \cdot F^{\phi}}=F^{\phi} \dot{S}_{o}^{e x 2}
$$


Equation (10) shows that the optimal operating point corresponds to the case where the load resistance defined by $F_{\text {load }}^{\phi}=\frac{\dot{W}}{\dot{S}^{2}}$ is equal to the internal resistance $F^{\varphi}$. This is again a well-known result in the field of thermoelectric conversion [46]. The optimal electric current is defined by: $I_{o}^{\mathrm{ex}}=\frac{\dot{S}_{o}^{\mathrm{ex}}}{\alpha}$ where $\alpha$ is the Seebeck coefficient.

The energy conversion efficiency of the exo-reversible heat engine at the optimal operating point is lower than the Carnot efficiency:

$$
\eta_{o}^{\mathrm{ex}}=\frac{\eta_{c}}{1+f_{c}+\left(1-\eta_{c}\right) f_{h}+4 \frac{K_{\lambda} F^{\varphi}}{T_{h s}}}<\eta_{c}
$$

It should be noted that in the case of an exo-reversible heat engine, the internal thermal conductance $K_{\lambda}$ does not affect the maximum output power but the associated energy conversion efficiency (cf. right-hand diagram on Figure 4).

From Figure 2, we can deduce the expressions of the rate of entropy generation related to internal dissipation $\dot{\sigma}_{\varphi}^{e x}$ and heat leakage $\dot{\sigma}_{\lambda}^{e x}$ in terms of entropy flow rate $\dot{S}$ :

$$
\begin{gathered}
\dot{\sigma}_{\varphi}^{e x}=\dot{S}_{\varphi h}+\dot{S}_{\varphi c}=\frac{f_{h} \Phi}{T_{h s}}+\frac{f_{c} \Phi}{T_{c s}}=\left(\frac{f_{h}}{T_{h s}}+\frac{f_{c}}{T_{c s}}\right) F^{\varphi} \dot{S}^{2} \\
\dot{\sigma}_{\lambda}^{e x}=\dot{S}_{\lambda c}-\dot{S}_{\lambda h}=\frac{\dot{Q}_{\lambda}}{T_{c s}}-\frac{\dot{Q}_{\lambda}}{T_{h s}}=K_{\lambda} \frac{\left(T_{h s}-T_{c s}\right)^{2}}{T_{c s} T_{h s}}
\end{gathered}
$$

The rate of entropy generation related to internal dissipation $\dot{\sigma}_{\varphi}^{e x}$ is proportional to the square of the entropy flow rate involved in the energy conversion $\dot{S}$. At point (A) defined by a zero entropy flow rate, this term is thus zero ( $c f$. Figure 5). The rate of entropy generation related to heat leakage $\dot{\sigma}_{\lambda}^{e x}$ is constant. We can then express the rate of total entropy generation $\dot{\sigma}^{e x}$ within the exo-reversible heat engine in the form:

$$
\dot{\sigma}^{e x}=\dot{\sigma}_{\lambda}^{e x}+\dot{\sigma}_{\varphi}^{e x}=\dot{\sigma}_{\lambda}^{e x}(1+\xi) \text { with } \xi=\frac{\dot{\sigma}_{\varphi}^{e x}}{\dot{\sigma}_{\lambda}^{e x}}=\frac{\left(f_{h} T_{c s}+f_{c} T_{h s}\right) F^{\varphi}}{K_{\lambda}\left(T_{h s}-T_{c s}\right)^{2}} \dot{S}^{2}
$$

Figure 5. Rates of entropy generation within an exo-reversible heat engine.

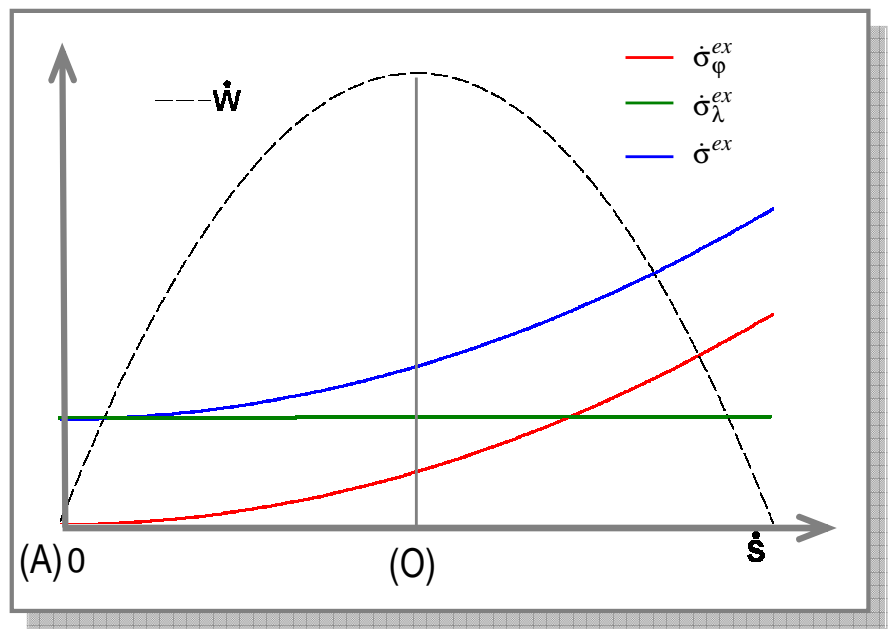


At point $(\mathrm{O})$, the ratio $\xi_{o}$ is equal to $\frac{1}{4} \cdot \frac{\left(f_{h} T_{c s}+f_{c} T_{h s}\right)}{K_{\lambda} F^{\varphi}}$ which in the case of a thermoelectric conversion, represents a quarter of the figure of merit ZT at temperature $\left(f_{h} T_{c s}+f_{c} T_{h s}\right)$.

\section{Modeling of an Irreversible Heat Engine at Steady State}

Figure 6 shows the Bond-Graph diagram at steady state of an irreversible heat engine which is built from the exo-reversible heat engine detailed in the previous section, by adding the finite constraint of the conductances connecting the heat engine to heat source and heat sink. As above, we assume that the overall conductances of the heat exchangers $K_{h}$ and $K_{c}$ are constant.

Figure 6. Bond-Graph diagram of an irreversible heat engine at steady state.

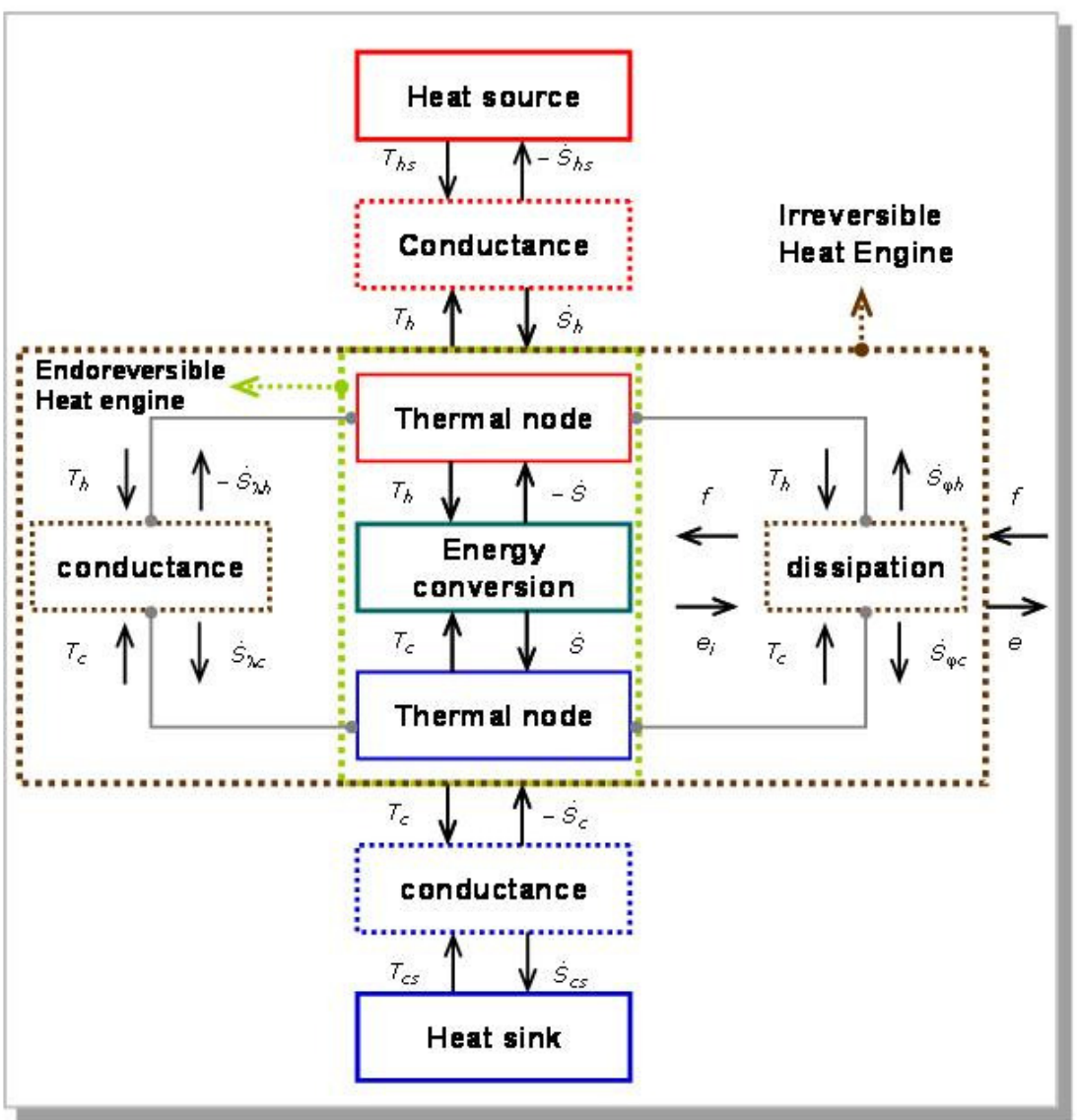

\subsection{Power Balance at Steady State}

From Figures 6 and 7, the power balance can be written as:

$$
\begin{aligned}
& \dot{Q}_{h}=\dot{q}_{h}+\dot{Q}_{\lambda}-f_{h} \Phi=\dot{S} T_{h}+\dot{Q}_{\lambda}-f_{h} F^{\varphi} \dot{S}^{2} \\
& \dot{Q}_{c}=\dot{q}_{c}+\dot{Q}_{\lambda}+f_{c} \Phi=\dot{S} T_{c}+\dot{Q}_{\lambda}+f_{c} F^{\varphi} \dot{S}^{2}
\end{aligned}
$$

with the laws of heat transfer:

$$
\dot{Q}_{h}=K_{h}\left(T_{h s}-T_{h}\right), \dot{Q}_{c}=K_{c}\left(T_{c}-T_{c s}\right), \dot{Q}_{\lambda}=K_{\lambda}\left(T_{h}-T_{c}\right)
$$


The output mechanical power can thus be written as (Figure 6):

$$
\dot{W}=\dot{Q}_{h}-\dot{Q}_{c}=\left(\dot{q}_{h}-\dot{q}_{c}\right)-\Phi=\dot{S}\left(T_{h}-T_{c}\right)-F^{\varphi} \dot{S}^{2}
$$

note that the expression (16) does not enable to give the function $\dot{W}(\dot{S})$ which is requisite to plot the operating curve of the heat engine, because the temperatures $T_{h}$ and $T_{c}$ depend implicitly on the operating point, as we shall see below.

Figure 7. Energy balance of an irreversible heat engine.

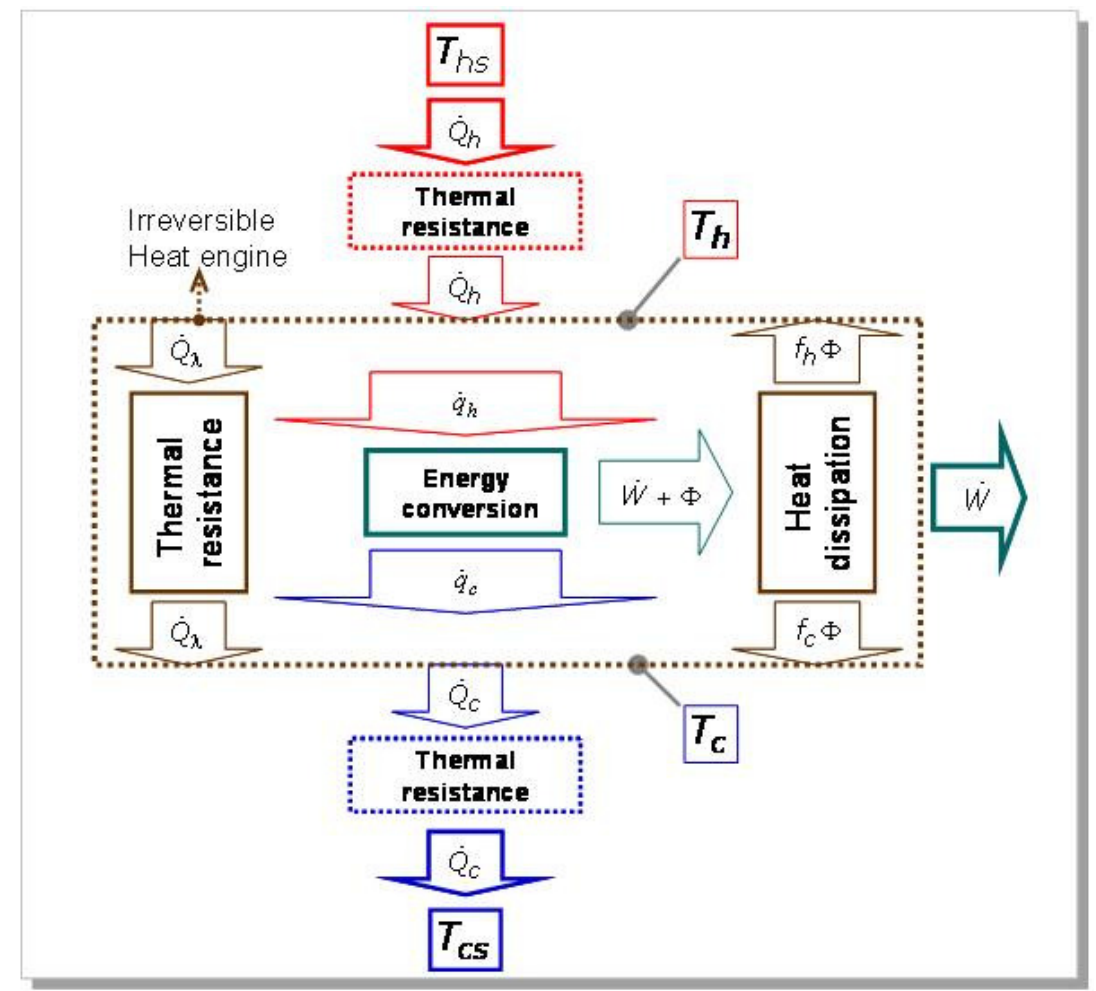

\subsection{Analytical Expressions of the Operating Point}

By eliminating the thermal powers $\dot{Q}_{h}, \dot{Q}_{c}$ and $\dot{Q}_{\lambda}$, between the laws of heat transfer (15) and the thermal energy balances (14), we obtain a linear system of two equations with temperatures $T_{c}$ and $T_{h}$ as unknowns and the entropy flow rate $\dot{S}$ as a parameter which is similar to that in [47]:

$$
\begin{gathered}
A(\dot{S}) \cdot T=B(\dot{S}) \text {, with } T=\left[\begin{array}{c}
T_{h} \\
T_{c}
\end{array}\right] \\
\text { and } A(\dot{S})=\left[\begin{array}{cc}
K_{h}+K_{\lambda}+\dot{S} & -K_{\lambda} \\
-K_{\lambda} & K_{c}+K_{\lambda}-\dot{S}
\end{array}\right], B(\dot{S})=\left[\begin{array}{c}
K_{h} T_{h s}+f_{h} F^{\varphi} \dot{S}^{2} \\
K_{c} T_{c s}+f_{c} F^{\varphi} \dot{S}^{2}
\end{array}\right]
\end{gathered}
$$

from the solution of the linear system (17) which expresses the temperatures $T_{c}$ and $T_{h}$ as a function of the entropy flow rate $\dot{S}$, we can calculate all the thermal powers from the laws of heat transfer (15), which allows us to express the output mechanical power based on the entropy flow rate (16). 


\subsection{Analysis of Effects of Internal Dissipation}

We assume here that the internal thermal conductance $K_{\lambda}$ is zero (no heat leakage within the heat engine). In this case, the matrix of the linear system (17) is diagonal. The solution is immediate:

$$
T_{h}(\dot{S})=\frac{K_{h} T_{h s}+f_{h} F^{\varphi} \dot{S}^{2}}{K_{h}+\dot{S}}, T_{c}(\dot{S})=\frac{K_{c} T_{c s}+f_{c} F^{\varphi} \dot{S}^{2}}{K_{c}-\dot{S}}
$$

by injecting the expressions of (18) in Equations (15) and (16), we obtain the expressions of the thermal powers $\dot{Q}_{h}, \dot{Q}_{c}$ and the output mechanical power $\dot{W}$ as a function of the entropy flow rate $\dot{S}$ :

$$
\begin{gathered}
\dot{Q}_{h}(\dot{S})=\frac{K_{h} T_{h s} \dot{S}-K_{h} f_{h} F^{\varphi} \dot{S}^{2}}{K_{h}+\dot{S}}, \dot{Q}_{c}(\dot{S})=\frac{K_{c} T_{c S} \dot{S}+K_{c} f_{c} F^{\varphi} \dot{S}^{2}}{K_{c}-\dot{S}} \\
\dot{W}(\dot{S})=\left(\frac{K_{h}\left(T_{h s}+K_{h} f_{h} F^{\varphi}\right)}{K_{h}+\dot{S}}-\frac{K_{c}\left(T_{c s}+K_{c} f_{c} F^{\varphi}\right)}{K_{c}-\dot{S}}\right) \dot{S}-\left(K_{h} f_{h}-K_{c} f_{c}\right) F^{\varphi} \dot{S}
\end{gathered}
$$

The nodal approach used here does not enable the distribution of the internal dissipated power $\Phi$ between the hot side $\left(f_{h}\right)$ and the cold side $\left(f_{c}\right)$ to be determined. The simplest hypothesis (and also the most widely used) is to divide this dissipated power equally between the hot side and the cold side $\ll f_{h}=f_{c}=\frac{1}{2} »$. However, given the arbitrary nature of this hypothesis, we prefer another hypothesis which allows us to cancel the last term in expression (19). Given the implicit relation $« f_{c}+f_{h}=1 »$, we obtain:

$$
K_{h} f_{h}=K_{c} f_{c}=K_{s c} \text { with } K_{s c}=\frac{K_{c} K_{h}}{K_{c}+K_{h}}
$$

The conductance $K_{s c}$ of Equation (20) corresponds to the configuration where the two resistances $1 / K_{h}$ and $1 / K_{c}$ are arranged in series. In the particular case where the two conductances $K_{c}$ and $K_{h}$ are equal, we have $K_{c}=K_{h}=2 K_{s c}$ which leads to $" f_{h}=f_{c}=\frac{1}{2} »$. Finally, we obtain an expression of the output mechanical power similar to that of the case of an endoreversible heat engine [1] where the temperatures of the heat source and the heat sink are virtually increased by the same amount [Equation (22)]:

$$
\begin{gathered}
\qquad \dot{W}(\dot{S})=\left(\frac{K_{h} T_{h s}^{\varphi}}{K_{h}+\dot{S}}-\frac{K_{c} T_{c s}^{\varphi}}{K_{c}-\dot{S}}\right) \dot{S} \text { with: } \\
T_{h s}^{\varphi}=T_{h s}+\varphi \cdot T_{s c} \text { and } T_{c s}^{\varphi}=T_{c s}+\varphi \cdot T_{s c} \\
\text { with } \varphi=\frac{K_{s c} F^{\varphi}}{T_{s c}} \text { (dimensionless number) }
\end{gathered}
$$

The expression of the output mechanical power (21) is analogous to the case of an endoreversible heat engine on condition that we work with the "apparent" temperatures of heat source and heat sink given by (22). In this case, it concerns an upward translation of these temperatures $\left(T_{h s}^{\varphi} \geq T_{h s}, T_{c s}^{\varphi} \geq T_{c s}\right.$ and $T_{h s}^{\varphi}-T_{c s}^{\varphi}=T_{h s}-T_{c s}$ ). 
We immediately obtain the cutoff entropy flow rate $\dot{S}_{s c}^{\varphi}$, a nontrivial solution of $\dot{W}(\dot{S})=0$ :

$$
\dot{S}_{s c}^{\varphi}=\frac{\dot{Q}_{s c}}{T_{s c}^{\varphi}}
$$

with $\dot{Q}_{s c}=K_{s c}\left(T_{h s}-T_{c s}\right), T_{s c}=\frac{K_{c} T_{c s}+K_{h} T_{h s}}{K_{c}+K_{h}}$ and $T_{s c}^{\varphi}=(1+\varphi) \cdot T_{s c}$

The internal dissipation increases the cutoff temperature compared to the case of the endoreversible heat engine but does not modify the heat flow rate [cf. Equation (23)]. As a result, the internal dissipation reduces the range of variation of the entropy flow rate $\dot{S}$ ( $c f$. Figure 8).

Figure 8. Deformation of the operating curve of heat engine in the presence of internal dissipation.
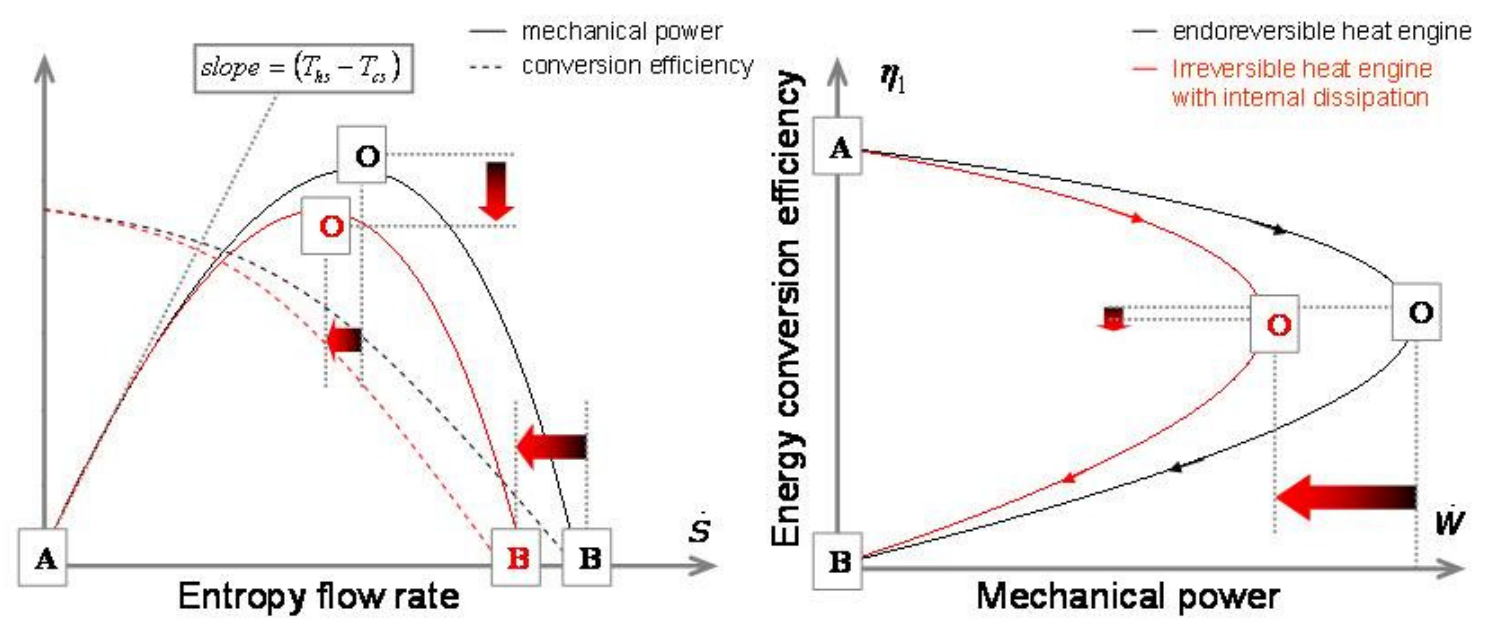

By deriving the expression (21) regarding the entropy flow rate $\dot{S}$, we obtain an expression similar to that obtained in the case of an endoreversible heat engine [1] where the temperatures of the heat source and the heat sink have been replaced by their apparent values [ $c f$. Equation (22)]:

$$
\frac{d \dot{W}}{d \dot{S}}=\left(\frac{K_{h} \sqrt{T_{h s}^{\varphi}}}{K_{h}+\dot{S}}\right)^{2}-\left(\frac{K_{c} \sqrt{T_{c S}^{\varphi}}}{K_{c}-\dot{S}}\right)^{2}
$$

we immediately deduce the expression of the optimal entropy flow rate:

$$
\dot{S}_{o}^{\varphi}=\frac{K_{c} K_{h}\left(\sqrt{T_{h s}^{\varphi}}-\sqrt{T_{c s}^{\varphi}}\right)}{K_{c} \sqrt{T_{c s}^{\varphi}}+K_{h} \sqrt{T_{h s}^{\varphi}}}
$$

Finally, by injecting $\dot{S}_{o}^{\varphi}$ in (21), we obtain the expression of the maximum output mechanical power by using the apparent temperatures $T_{h s}^{\varphi}$ and $T_{c s}^{\varphi}$ :

$$
\dot{W}_{o}^{\varphi}=K_{s c}\left(\sqrt{T_{h s}^{\varphi}}-\sqrt{T_{c s}^{\varphi}}\right)^{2}
$$


we can show that despite the conservation of the difference of temperatures $\left(T_{h s}^{\varphi}-T_{c s}^{\varphi}=T_{h s}-T_{c s}\right)$, the "thermal potential" $\Theta^{\varphi}=\left(\sqrt{T_{h s}^{\varphi}}-\sqrt{T_{c s}^{\varphi}}\right)^{2}$ involved in the expression of the maximum power (26) is lower than that of the endoreversible heat engine $\Theta=\left(\sqrt{T_{h s}}-\sqrt{T_{c s}}\right)^{2}$. In fact, by making a first-order Taylor expansion of $\Theta^{\varphi}$ with respect to the term $\varphi T_{s c}$, we obtain the following expression:

$$
\Theta^{\varphi} \approx \Theta-\varphi T_{s c}\left(\sqrt{\frac{T_{c s}}{T_{h s}}}+\sqrt{\frac{T_{h s}}{T_{c s}}}-2\right)
$$

By studying the direction of the variation of the function $f(x)=x+\frac{1}{x}-2$ where $x=\sqrt{\frac{T_{c s}}{T_{h s}}}$ in the range $[0,1]$, we can easily demonstrate the inequality $\sqrt{\frac{T_{c s}}{T_{h s}}}+\sqrt{\frac{T_{h s}}{T_{c s}}}-2 \geq 0$.

Moreover, the derivative of the output mechanical power (24) at the origin point is equal to the difference of temperatures of thermostats. According to the expressions of (22), we obtain the following relationship: $T_{h s}^{\varphi}-T_{c s}^{\varphi}=T_{h s}-T_{c s}$ which shows that the slope at the origin of mechanical power is not affected by the internal dissipation. Similarly, one can demonstrate that the slope at the origin of the thermal power $\left.\frac{\partial \dot{Q}_{h}}{\partial \dot{S}}\right|_{\dot{s}=0}=T_{h s}$ [Equation (19)] is not affected by the dissipation either. This explains why the energy conversion efficiency of the machine at $\dot{S}=0$ is equal to the Carnot efficiency $\eta_{1(\dot{S}=0)}=\frac{\left.\frac{\partial \dot{W}}{\partial \dot{S}}\right|_{\dot{s}=0}}{\left.\frac{\partial \dot{Q}_{h}}{\partial \dot{S}}\right|_{\dot{s}=0}}=\eta_{c}(c f$. Figure 8).

It remains to study the impact of the internal dissipation on the energy conversion efficiency. From Equation (19), we obtain the energy conversion efficiency as a function of the entropy flow rate $\dot{S}$ :

$$
\eta_{1}(\dot{S})=1-\left(\frac{K_{h}+\dot{S}}{K_{c}-\dot{S}}\right)\left(\frac{K_{c} T_{c s}+\varphi T_{s c} \dot{S}}{K_{h} T_{h s}-\varphi T_{s c} \dot{S}}\right)
$$

At point (A) of zero entropy flow rate, the energy conversion efficiency is equal to the Carnot efficiency, as shown above. Therefore, one should not use the apparent Carnot efficiency $1-\frac{T_{c s}^{\varphi}}{T_{h s}^{\varphi}}$ to calculate the energy conversion efficiency at point (A).

At point (B) where the entropy flow rate is equal to the cutoff entropy flow rate $\dot{S}_{s c}^{\varphi}$ given by the expression of (23), the conversion efficiency is naturally zero. For the optimal point $(\mathrm{O})$, by injecting the optimal entropy flow rate $\dot{S}_{o}^{\varphi}$ in the expression (28), we obtain:

$$
\eta_{o}^{\varphi}=\eta_{C N C A}^{\varphi} \frac{\sqrt{T_{h s}^{\varphi}}}{\sqrt{T_{h s}^{\varphi}}-G^{\varphi}} \text { with } \eta_{C N C A}^{\varphi}=1-\sqrt{\frac{T_{c s}^{\varphi}}{T_{h s}^{\varphi}}} \text { and } G^{\varphi}=\frac{K_{c} K_{h} F^{\varphi}}{K_{c} \sqrt{T_{c s}^{\varphi}}+K_{h} \sqrt{T_{h s}^{\varphi}}}
$$

It may be noted that this associated efficiency differs from the apparent CNCA efficiency obtained by using the apparent temperatures of heat source $T_{h s}^{\varphi}$ and heat sink $T_{c s}^{\varphi}\left(\eta_{o}^{\varphi} \geq \eta_{C N C A}^{\varphi}\right)$.

In addition, one should note that contrary to the endoreversible heat engine, the optimal efficiency $\eta_{o}^{\varphi}$ associated to the maximum power depends not only on the temperatures of heat source and heat sink but also the conductances $K_{c}, K_{h}$ and the internal "resistance" $F^{\varphi}$. 
Finally the operating curve of the heat engine in the presence of internal dissipation in the $\left[\dot{W}, \eta_{1}\right]$ diagram deforms according to the diagram on the right of Figure 8.

\subsection{Analysis of Effects of Internal Heat Leakage}

We will now analyze the effect of the internal heat transfer on the performance of the machine $\left(K_{\lambda}>0\right)$. We assume here that there is no internal dissipation $\left(F^{\varphi}=0\right)$. According to the linear system (17), the internal heat transfer creates a coupling term between the temperatures $T_{c}$ and $T_{h}$ [non-zero terms on the diagonal of matrix A (17)]. To solve this system, we will first simplify the matrix and calculate the determinant of the matrix in the form:

$$
\Delta(\dot{S})=\left(K_{h}^{\lambda}+\dot{S}\right)\left(K_{c}^{\lambda}-\dot{S}\right)
$$

A simple calculation gives us the apparent conductances $K_{c}^{\lambda}$ and $K_{h}^{\lambda}$ by reference to the endoreversible heat engine [1]:

$$
K_{h}^{\lambda}=K_{h}+K_{\lambda}^{\lambda}, K_{c}^{\lambda}=K_{c}+K_{\lambda}^{\lambda}, \text { with } K_{\lambda}^{\lambda}=\frac{K_{c}+K_{h}}{2}[\sqrt{1+2 \lambda}-1] \text { and } \lambda=\frac{2 K_{\lambda}}{K_{c}+K_{h}}
$$

The inversion of the linear system (17) then makes it possible to express the temperatures $T_{c}$ and $T_{h}$ in terms of $\dot{S}$ :

$$
\begin{aligned}
& T_{h}(\dot{S})=\frac{\left(K_{c}-\dot{S}\right) K_{h} T_{h s}+K_{\lambda}\left(K_{c} T_{c S}+K_{h} T_{h s}\right)}{\left(K_{h}^{\lambda}+\dot{S}\right)\left(K_{c}^{\lambda}-\dot{S}\right)} \\
& T_{c}(\dot{S})=\frac{\left(K_{h}+\dot{S}\right) K_{c} T_{c S}+K_{\lambda}\left(K_{c} T_{c S}+K_{h} T_{h s}\right)}{\left(K_{h}^{\lambda}+\dot{S}\right)\left(K_{c}^{\lambda}-\dot{S}\right)}
\end{aligned}
$$

We can now express the output mechanical power as a function of the entropy flow rate $\dot{S}$ from the relations (32) and (16) which becomes here $\dot{W}=\left(T_{h}-T_{c}\right) \dot{S}$ (under the assumption $F^{\varphi}=0$ ):

$$
\begin{gathered}
\dot{W}(\dot{S})=\frac{1}{\sqrt{1+2 \lambda}}\left(\frac{K_{h}^{\lambda} T_{h s}^{\lambda}}{K_{h}^{\lambda}+\dot{S}}-\frac{K_{c}^{\lambda} T_{c s}^{\lambda}}{K_{c}^{\lambda}-\dot{S}}\right) \dot{S} \\
\text { with: } T_{h s}^{\lambda}=\frac{K_{h} T_{h s}+K_{\lambda}^{\lambda} T_{s c}}{K_{h}+K_{\lambda}^{\lambda}}, T_{c s}^{\lambda}=\frac{K_{c} T_{c s}+K_{\lambda}^{\lambda} T_{s c}}{K_{c}+K_{\lambda}^{\lambda}}
\end{gathered}
$$

As in the previous case, we again obtain the apparent temperatures of heat source and heat sink, which brings us back to the case of the endoreversible heat engine. However, it should be noted that the expression (33) has a factor $\frac{1}{\sqrt{1+2 \lambda}}$ which is equal to 1 in the case of the endoreversible heat engine.

Unlike the previous case (analysis of effects of internal dissipation), we observe here an "apparent" increase in the temperature of the heat sink and an "apparent" decrease in the temperature of the heat source and finally an "apparent" decrease in the pinch between heat source and heat sink:

$$
T_{c s}^{\lambda} \geq T_{c s} T_{h s}^{\lambda} \leq T_{h s}, \rightarrow T_{h s}^{\lambda}-T_{c s}^{\lambda} \leq T_{h s}-T_{c s}
$$

which reflects a predictable degradation of the output mechanical power compared to the case of an endoreversible heat engine.

From the expression of (33), one can determine the operating range of the machine by calculating 
the cutoff entropy flow rate $\dot{S}_{s c}^{\lambda}$, a nontrivial solution of the equation $\dot{W}(\dot{S})=0$ :

$$
\begin{gathered}
\dot{S}_{s c}^{\lambda}=\frac{\dot{Q}_{s c}^{\lambda}}{T_{s c}^{\lambda}} \\
\text { with: } \dot{Q}_{s c}^{\lambda}=\frac{K_{c}^{\lambda} K_{h}^{\lambda}\left(T_{h s}^{\lambda}-T_{c s}^{\lambda}\right)}{K_{c}^{\lambda}+K_{h}^{\lambda}} \text { and } T_{s c}^{\lambda}=\frac{K_{c}^{\lambda} T_{c s}^{\lambda}+K_{h}^{\lambda} T_{h s}^{\lambda}}{K_{c}^{\lambda}+K_{h}^{\lambda}}
\end{gathered}
$$

We can easily demonstrate the following equalities from (31):

$$
T_{s c}^{\lambda}=\frac{T_{s c}}{\sqrt{1+2 \lambda}}, \dot{Q}_{s c}^{\lambda}=\frac{\dot{Q}_{s c}}{\sqrt{1+2 \lambda}} \rightarrow \dot{S}_{s c}^{\lambda}=\dot{S}_{s c}
$$

We conclude that the cutoff entropy flow rate is not affected by the internal heat leakage even though it corresponds to a smaller cutoff thermal power $\left(\dot{Q}_{s c}^{\lambda} \leq \dot{Q}_{s c}\right)$. This confirms the interest of selecting the entropy flow rate $\dot{S}$ involved in reversible energy conversion as the control variable of the heat engine.

By deriving the mechanical power (33) regarding the entropy flow rate $\dot{S}$, we again obtain an analogous expression by reference to the case of an endoreversible heat engine by using apparent conductances and apparent temperatures (with a factor $\frac{1}{\sqrt{1+2 \lambda}}$ ):

$$
\frac{d \dot{W}}{d \dot{S}}=\frac{1}{\sqrt{1+2 \lambda}}\left(\left(\frac{K_{h}^{\lambda} \sqrt{T_{h s}^{\lambda}}}{K_{h}^{\lambda}+\dot{S}}\right)^{2}-\left(\frac{K_{c}^{\lambda} \sqrt{T_{c s}^{\lambda}}}{K_{c}^{\lambda}-\dot{S}}\right)^{2}\right)
$$

which makes it possible to calculate the optimal entropy flow rate $\dot{S}_{o}^{\lambda}$ [solution of (38)]:

$$
\dot{S}_{o}^{\lambda}=\frac{K_{c}^{\lambda} K_{h}^{\lambda}\left(\sqrt{T_{h s}^{\lambda}}-\sqrt{T_{c s}^{\lambda}}\right)}{K_{c}^{\lambda} \sqrt{T_{c s}^{\lambda}}+K_{h}^{\lambda} \sqrt{T_{h s}^{\lambda}}}
$$

The expression of the optimal entropy flow rate is perfectly analogous to the case of an endoreversible heat engine when we use the "apparent" conductances and temperatures.

Finally, by injecting the optimal entropy flow rate $\dot{S}_{o}^{\lambda}$ in the expression of (33), we obtain the expression of the maximum output mechanical power $\dot{W}_{o}^{\lambda}$ as a function of different physical parameters:

$$
\dot{W}_{o}^{\lambda}=K_{s c}^{\lambda}\left(\sqrt{T_{h s}^{\lambda}}-\sqrt{T_{c s}^{\lambda}}\right)^{2} \text { with } K_{s c}^{\lambda}=K_{s c}+\frac{K_{\lambda}}{1+2 \lambda}\left(\frac{K_{c}-K_{h}}{K_{c}+K_{h}}\right)^{2}
$$

Given the inequality (35), we immediately deduce the following inequality $\Theta^{\lambda} \leq \Theta$ where $\Theta^{\lambda}$ is defined by $\Theta^{\lambda}=\left(\sqrt{T_{h s}^{\lambda}}-\sqrt{T_{c s}^{\lambda}}\right)^{2}$. Finally, we have two contradictory effects: a dominant effect related to the temperatures $\Theta^{\lambda}$ which degrades the mechanical power and a second effect of the overall thermal conductance which reduces the degradation $\left\langle K_{s c}^{\lambda} \geq K_{s c} »\right.$. In fact, from Equation (38) we prove that the slope at the origin point of the curve of mechanical power $\frac{d \dot{W}}{d \dot{S}}(\dot{S}=0)=\frac{T_{h s}^{\lambda}-T_{c s}^{\lambda}}{\sqrt{1+2 \lambda}} \leq T_{h s}-T_{c s}$ is lower than that of the endoreversible heat engine $\lambda=0$. And as the cutoff entropy flow rate is not modified by the internal heat leakage, the curve of mechanical power is reduced by the heat leakage ( $c f$. Figure 9 ), as is 
the maximum power $\left(\dot{W}_{o}^{\lambda} \leq \dot{W}_{o}\right)$.

By using the laws of heat transfer, we can express the energy conversion efficiency as a function of the temperatures $T_{c}(\dot{S})$ and $T_{h}(\dot{S})$ given by (32):

$$
\eta_{1}(\dot{S})=\frac{\dot{W}}{\dot{Q}_{h}}=\frac{T_{h}(\dot{S})-T_{c}(\dot{S})}{T_{h s}-T_{h}(\dot{S})} \cdot \frac{\dot{S}}{K_{h}}
$$

Figure 9. Deformation of the operating curve of heat engine in presence of internal heat leakage in the case where $K_{h} \geq K_{c}$.

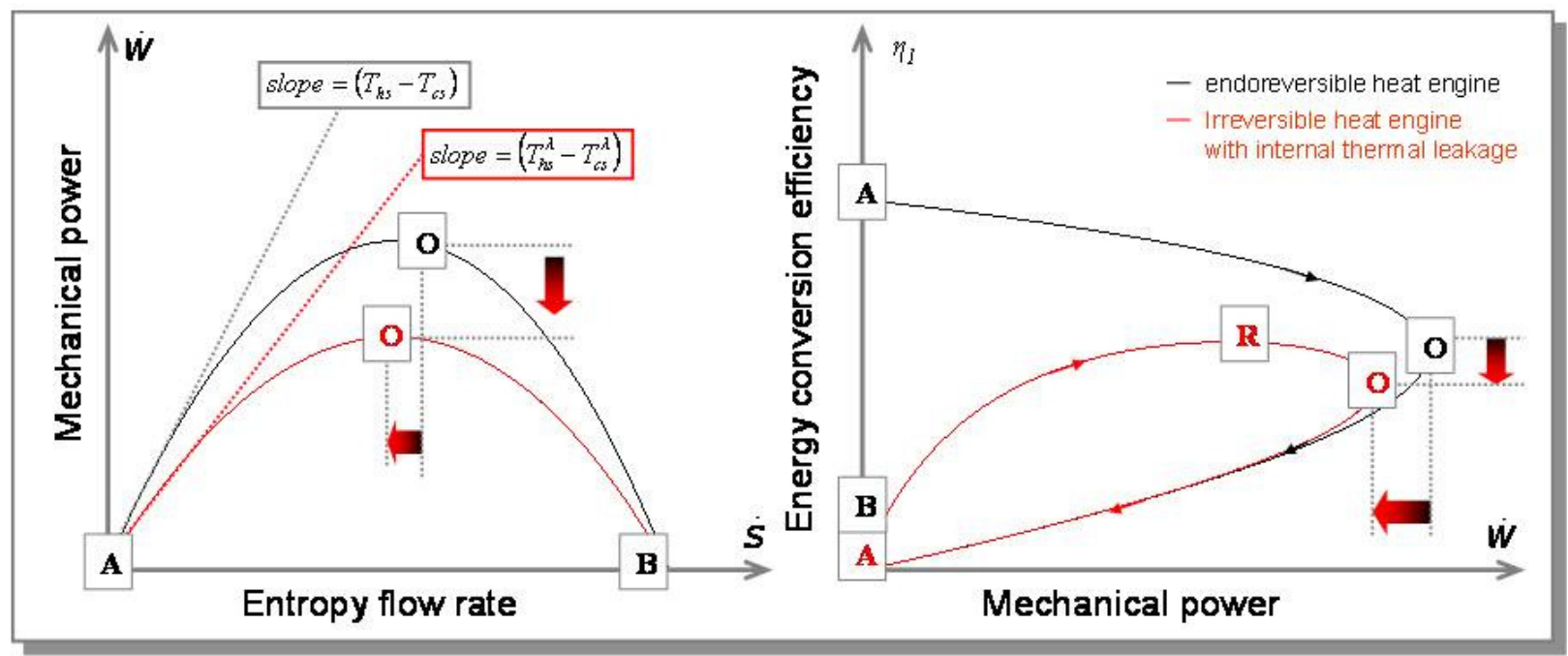

At point (A) defined by a zero entropy flow rate, the thermal power received by the engine from the heat source is equal to the internal heat loss: $\dot{Q}_{h}=\dot{Q}_{\lambda}[c f$. Equation (14)]. We can thus write at the point (A) the following relationship with the assumption $K_{\lambda}>0: \eta_{1}(\dot{S} \approx 0) \approx \frac{\dot{W}}{\dot{Q}_{\lambda}}=\frac{\dot{S}\left(T_{h}-T_{c}\right)}{K_{\lambda}\left(T_{h}-T_{c}\right)}=\frac{\dot{S}}{K_{\lambda}}$. As a result, unlike the case of an endoreversible heat engine, even the case including internal dissipation, the energy conversion efficiency at point (A) in presence of internal heat leakage is zero. The heat engine behaves at this point as three thermal resistances in series with the only effect that the thermal power $\dot{Q}_{h}=\dot{Q}_{c}=\dot{Q}_{\lambda}$ is transferred from heat source to heat sink without any production of mechanical power.

The right-hand diagram on Figure 9 shows the deformation of the operating curve of the heat engine in presence of internal heat leakage in $\left[\dot{W}, \eta_{1}\right]$. As the energy conversion efficiency is zero at point $(\mathrm{A})$, there exists a point $(\mathrm{R})$ between $(\mathrm{A})$ and $(\mathrm{O})$ for which the energy conversion efficiency is maximum. As a result, the optimum operating range is limited by the portion $[R, O][48,49]$.

\subsection{Analysis of Combined Effects of Internal Dissipation and Heat Leakage}

Here we have a nonzero leakage conductance $K_{\lambda}$ and a nonzero dissipation coefficient $F^{\varphi}$. By applying the same approach as for the previous two cases, we again obtain an expression of the output mechanical power similar to the case of the endoreversible heat engine:

$$
\dot{W}=\frac{1}{\sqrt{1+2 \lambda}}\left(\frac{K_{h}^{\lambda} T_{h s}^{\lambda, \varphi}}{K_{h}^{\lambda}+\dot{S}}-\frac{K_{c}^{\lambda} T_{c s}^{\lambda, \varphi}}{K_{c}^{\lambda}-\dot{S}}\right) \dot{S} \text { with: }
$$




$$
\begin{gathered}
T_{h s}^{\lambda, \varphi}=T_{h s}^{\lambda}+\left(\varphi+\xi^{\lambda, \varphi}\right) T_{s c}, T_{c s}^{\lambda, \phi}=T_{c s}^{\lambda}+\left(\varphi+\xi^{\lambda, \varphi}\right) T_{s c} \\
\text { with: } \xi^{\lambda, \varphi}=\frac{K_{\lambda} F^{\varphi}}{T_{s c}} \text { (dimensionless number) }
\end{gathered}
$$

We see here that the apparent temperatures depend on the two parameters $\lambda$ and $\varphi$ with a coupling term given by the dimensionless number $\xi^{\lambda, \varphi}\left(\xi^{\lambda, \varphi}=\lambda \varphi \cdot \frac{\left(K_{h}+K_{c}\right)^{2}}{2 K_{h} K_{c}}\right)$. In the case of a thermoelectric conversion, we have $\xi^{\lambda, \varphi}=\frac{K_{\lambda} R}{\alpha^{2} T_{s c}}=\frac{1}{Z T_{s c}}$ which is the inverse of figure of merit of thermoelectric material at temperature $T_{s c}$.

The cutoff entropy flow rate $\dot{S}_{s c}^{\lambda, \varphi}$ is the nontrivial solution of (42):

$$
\begin{gathered}
\dot{S}_{s c}^{\lambda, \varphi}=\frac{\dot{Q}_{s c}^{\lambda, \varphi}}{T_{s c}^{\lambda, \varphi}} \\
\text { with: } \dot{Q}_{s c}^{\lambda, \varphi}=\frac{K_{c}^{\lambda} K_{h}^{\lambda}\left(T_{h s}^{\lambda, \varphi}-T_{c s}^{\lambda, \varphi}\right)}{K_{c}^{\lambda}+K_{h}^{\lambda}} \text { and } T_{s c}^{\lambda, \varphi}=\frac{K_{c}^{\lambda} T_{c s}^{\lambda, \varphi}+K_{h}^{\lambda} T_{h s}^{\lambda, \varphi}}{K_{c}^{\lambda}+K_{h}^{\lambda}}
\end{gathered}
$$

we can easily demonstrate the following relationship:

$$
\begin{gathered}
T_{s c}^{\lambda, \varphi}=T_{s c}^{\lambda}+\left(\varphi+\xi^{\lambda, \varphi}\right), T_{s c} \geq T_{s c}^{\lambda} \text { and } \dot{Q}_{s c}^{\lambda, \varphi}=\dot{Q}_{s c}^{\lambda} \\
\rightarrow \quad \dot{S}_{s c}^{\lambda, \varphi} \leq \dot{S}_{s c}^{\lambda}=\dot{S}_{s c}
\end{gathered}
$$

finally, by deriving expression (42) regarding the entropy flow rate $\dot{S}$, we obtain:

$$
\frac{d \dot{W}}{d \dot{S}}=\frac{1}{\sqrt{1+2 \lambda}}\left(\left(\frac{K_{h}^{\lambda} \sqrt{T_{h s}^{\lambda, \varphi}}}{K_{h}^{\lambda}+\dot{S}}\right)^{2}-\left(\frac{K_{c}^{\lambda} \sqrt{T_{c s}^{\lambda, \varphi}}}{K_{c}^{\lambda}-\dot{S}}\right)^{2}\right)
$$

we then have the expression of the optimal entropy flow rate $\dot{S}_{o}^{\lambda, \varphi}$ which is strictly analogous to the previous cases:

$$
\dot{S}_{o}^{\lambda, \varphi}=\frac{K_{c}^{\lambda} K_{h}^{\lambda}\left(\sqrt{T_{h s}^{\lambda, \varphi}}-\sqrt{T_{c s}^{\lambda, \varphi}}\right)}{K_{c}^{\lambda} \sqrt{T_{c s}^{\lambda, \varphi}}+K_{h}^{\lambda} \sqrt{T_{h s}^{\lambda, \varphi}}}
$$

Finally, by injecting this optimal entropy flow rate $\dot{S}_{o}^{\lambda, \varphi}$ in (42), we obtain the maximum output mechanical power $\dot{W}_{o}^{\lambda, \varphi}$ in terms of different physical parameters:

$$
\dot{W}_{o}^{\lambda, \varphi}=K_{s c}^{\lambda}\left(\sqrt{T_{h s}^{\lambda, \varphi}}-\sqrt{T_{c s}^{\lambda, \varphi}}\right)^{2}
$$

here we have also an analogous expression compared with the endoreversible heat engine provided that the apparent temperatures and the apparent overall conductance $K_{s c}^{\lambda}$ given by Equation (40) are used. Regarding the energy conversion efficiency, we combine the two effects of internal dissipation and heat transfer, and in particular a zero efficiency at point (A) as shown in Figure 10.

Figure 10 illustrates the separate and combined effects of internal dissipation and heat leakage on the operating curve of the irreversible heat engine. In particular, on the right-hand figure, we plotted the «CNCA » efficiencies obtained by using the apparent temperatures of heat source and heat sink 
(horizontal curves). In conclusion, these CNCA efficiencies are not comparable to the real optimal efficiencies except in the case of endoreversible heat engines.

Figure 10. Deformation of operating curves of heat engine in presence of internal dissipation alone (red); heat leakage alone (green) and combined effects (purple).

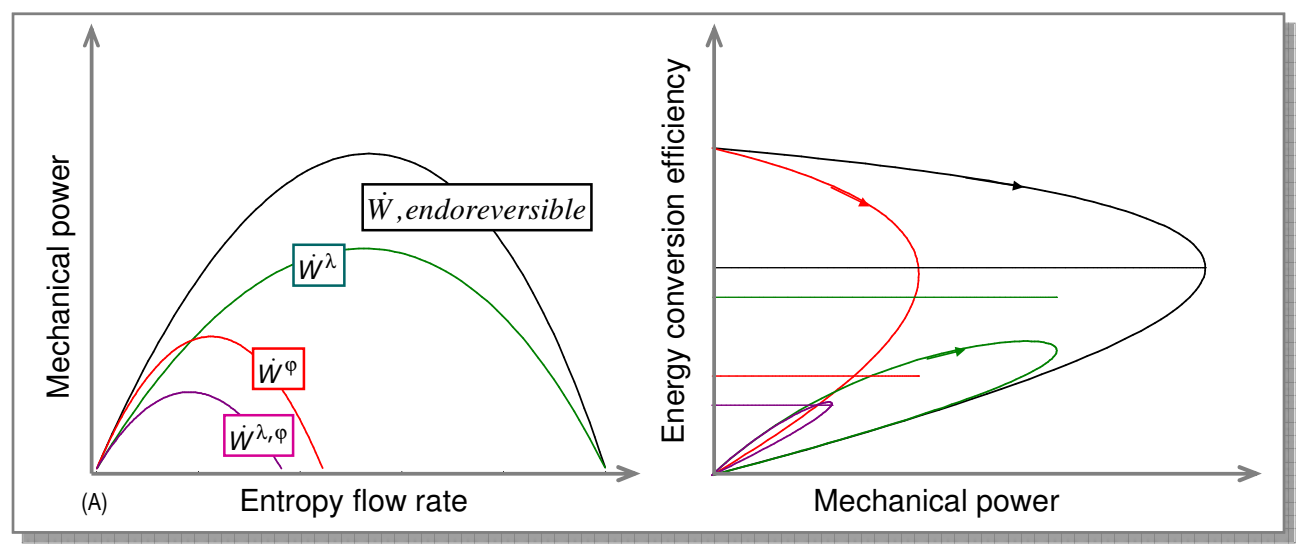

The parameter $\xi^{\lambda, \varphi}$ alone cannot be used to characterize the performance of a heat engine. For a given $\xi^{\lambda, \varphi}$, the maximum output power may be different according to the values of $\lambda$ and $\varphi$ (cf. Figure 11). As a result, in the case of thermoelectric conversion, we should not base all the research of thermoelectric materials only on the figure of merit ZT.

Figure 11. Deformation of operating curves for one given $\xi^{\lambda, \varphi}$.

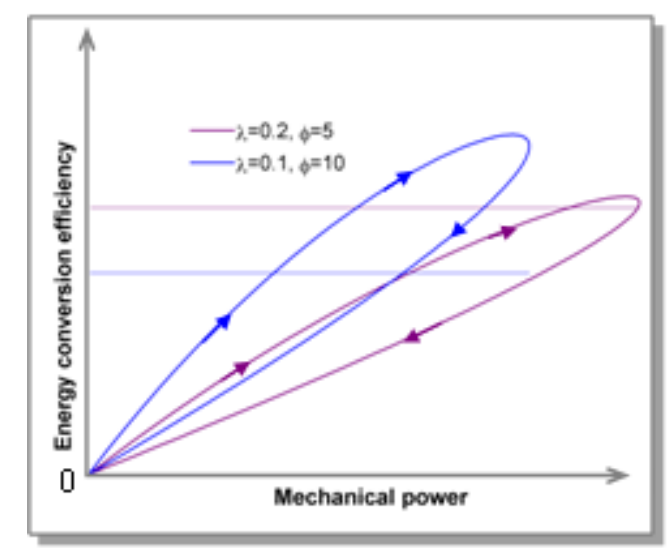

Starting from the irreversible heat engine detailed above ( $c f$. Figure 7), we add an external thermal conductance $K_{l}$ assumed constant to represent the heat leakage between heat source and heat sink (cf. Figure 12). 
Figure 12. Irreversible heat engine with external heat leakage between thermostats.

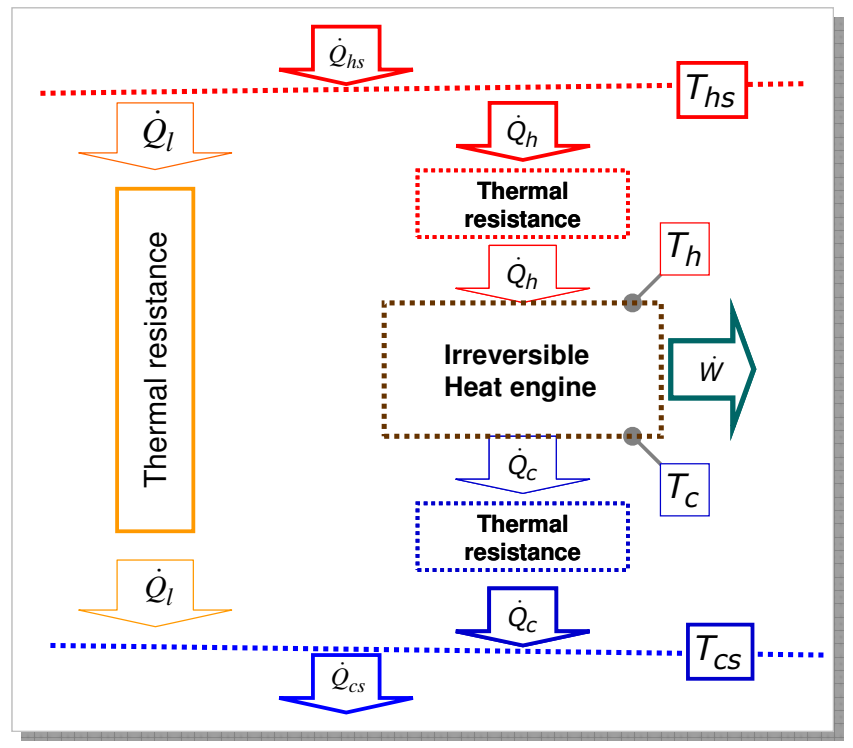

According to Figure 12, the power balance can be written as:

$$
\begin{gathered}
\dot{Q}_{h s}=\dot{Q}_{h}+\dot{Q}_{l}, \dot{Q}_{c s}=\dot{Q}_{c}+\dot{Q}_{l} \\
\rightarrow \dot{W}=\dot{Q}_{h s}-\dot{Q}_{c s}=\dot{Q}_{h}-\dot{Q}_{c}
\end{gathered}
$$

we note that the external heat leakage does not affect the mechanical power. The results obtained above concerning the expressions of optimal entropy flow rate $\dot{S}_{o}^{\lambda, \varphi}$, cutoff entropy flow rate $\dot{S}_{s c}^{\lambda, \varphi}$ and maximum output power $\dot{W}_{o}^{\lambda, \varphi}$ remain valid [cf. Equations (44), (47), (48)]. However, the external heat leakage reduces the overall energy conversion efficiency of the system defined as $\eta_{1}^{s y}(\dot{S})=\frac{\dot{W}(\dot{S})}{\dot{Q}_{h s}(\dot{S})}=\frac{\dot{W}(\dot{S})}{\dot{Q}_{h}(\dot{S})+\dot{Q}_{l}}(c f$. Figure 13).

Figure 13. Operating curves of irreversible heat engine.

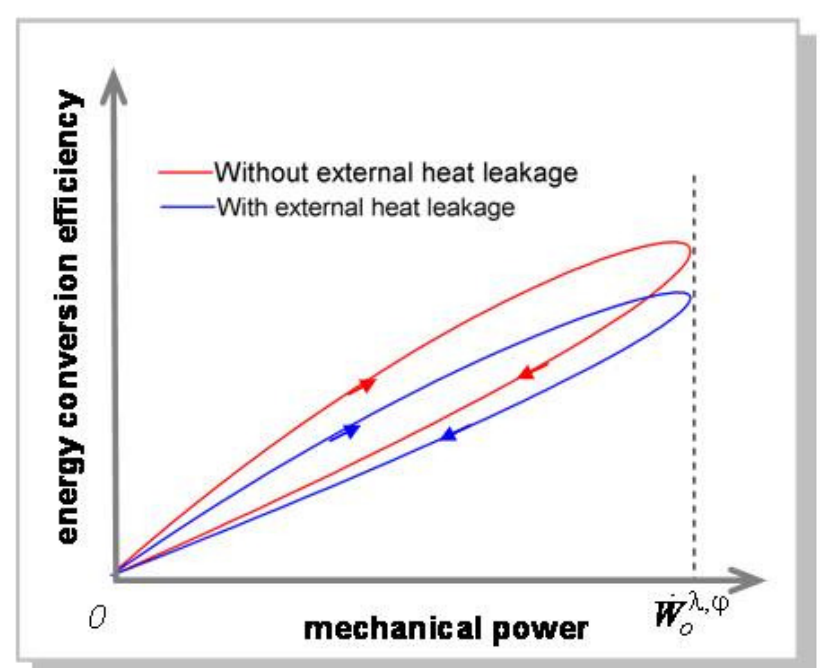




\subsection{Optimal Allocation of Conductances}

The maximum output mechanical power in the case of an Endoreversible Heat Engine is given by the following expression which is a product of two terms. The first term $K_{s c}$ is a function of the conductances $K_{h}$ and $K_{c}$, and the second term $\Theta$ is a function of temperatures:

$$
\dot{W}_{o}=K_{s c} \Theta, \quad K_{s c}=\frac{K_{c} K_{h}}{K_{c}+K_{h}}, \Theta=\left(\sqrt{T_{h s}}-\sqrt{T_{c s}}\right)^{2}
$$

The term $\Theta=\left(\sqrt{T_{h s}}-\sqrt{T_{c s}}\right)^{2}$ represents the effect of the temperatures of heat source and heat sink on the maximum output power. This term is considerably lower than the difference of temperatures $\Delta T=T_{h s}-T_{c s}$. For example, for $T_{c s}=300 \mathrm{~K}$ and $T_{h s}=900 \mathrm{~K}$, we have $\Delta T=600 \mathrm{~K}$ and $\Theta=161 \mathrm{~K}$ only!

We can explain the disparity between the factor $\Theta$ and the difference of temperatures $\Delta T$ by noting that the first one is proportional to the difference between the arithmetic average and the geometric average of the temperatures $T_{h s}$ and $T_{c s}$ :

$$
\Theta=2\left(\frac{T_{c s}+T_{h s}}{2}-\sqrt{T_{c s} T_{h s}}\right)
$$

For fixed temperatures $T_{h s}$ and $T_{c s}$, the single degree of freedom to increase the maximum output power is the constrained allocation of conductances $K_{h}$ and $K_{c}$. One can imagine different types of constraints such as the total heat exchange area allocated [23]. By designating the overall heat transfer coefficients $U_{h}$ and $U_{c}$, the total heat exchange area is given by:

$$
A=A_{c}+A_{h}=\frac{K_{c}}{U_{c}}+\frac{K_{h}}{U_{h}}
$$

to obtain the optimal allocation of conductances, we can use the method of Lagrange multipliers:

$$
\begin{gathered}
L=\frac{K_{c} K_{h}}{K_{c}+K_{h}} \Theta+\beta\left(\frac{K_{c}}{U_{c}}+\frac{K_{h}}{U_{h}}\right), \frac{\partial L}{\partial K_{c}}=0, \frac{\partial L}{\partial K_{h}}=0 \\
\rightarrow \frac{K_{c}}{\sqrt{U_{c}}}=\frac{K_{h}}{\sqrt{U_{h}}} \Leftrightarrow A_{c} \sqrt{U_{c}}=A_{h} \sqrt{U_{h}}
\end{gathered}
$$

In particular, if the heat exchange coefficients $U_{h}$ and $U_{c}$ are equal, the optimal allocation corresponds to the case of equipartition of conductances ( $c f$. Figure 14).

In presence of internal dissipation, the maximum output power is:

$$
\dot{W}_{o}^{\varphi}=K_{s c} \Theta^{\varphi}, \quad \Theta^{\varphi}=\left(\sqrt{T_{h s}+\varphi \cdot T_{s c}}-\sqrt{T_{c s}+\varphi \cdot T_{s c}}\right)^{2}
$$

In the special case where the optimization constraint is the total conductance $K_{c}+K_{h}=c s t$, we prove analytically (the calculations are heavy) that the internal dissipation has no effect on the optimal allocation of conductances by applying the method of Lagrange multipliers:

$$
L=K_{s c} \Theta^{\varphi}+\beta\left(K_{c}+K_{h}\right), \frac{\partial L}{\partial K_{c}}=0, \frac{\partial L}{\partial K_{h}}=0 \rightarrow K_{c}=K_{h}
$$


In presence of internal heat leakage, the maximum output power becomes:

$$
\begin{gathered}
\dot{W}_{o}^{\lambda}=K_{s c}^{\lambda} \Theta^{\lambda}, \quad \Theta^{\lambda}=\left(\sqrt{\frac{K_{h} T_{h s}+K_{\lambda}^{\lambda} T_{s c}}{K_{h}+K_{\lambda}^{\lambda}}}-\sqrt{\frac{K_{c} T_{h s}+K_{\lambda}^{\lambda} T_{s c}}{K_{c}+K_{\lambda}^{\lambda}}}\right)^{2} \\
\text { with: } K_{s c}^{\lambda}=K_{s c}+\frac{K_{\lambda}}{1+2 \lambda}\left(\frac{K_{c}-K_{h}}{K_{c}+K_{h}}\right)^{2}=\frac{K_{s c}+K_{\lambda}}{1+2 \lambda}
\end{gathered}
$$

Figure 14. Optimal allocation of conductances.

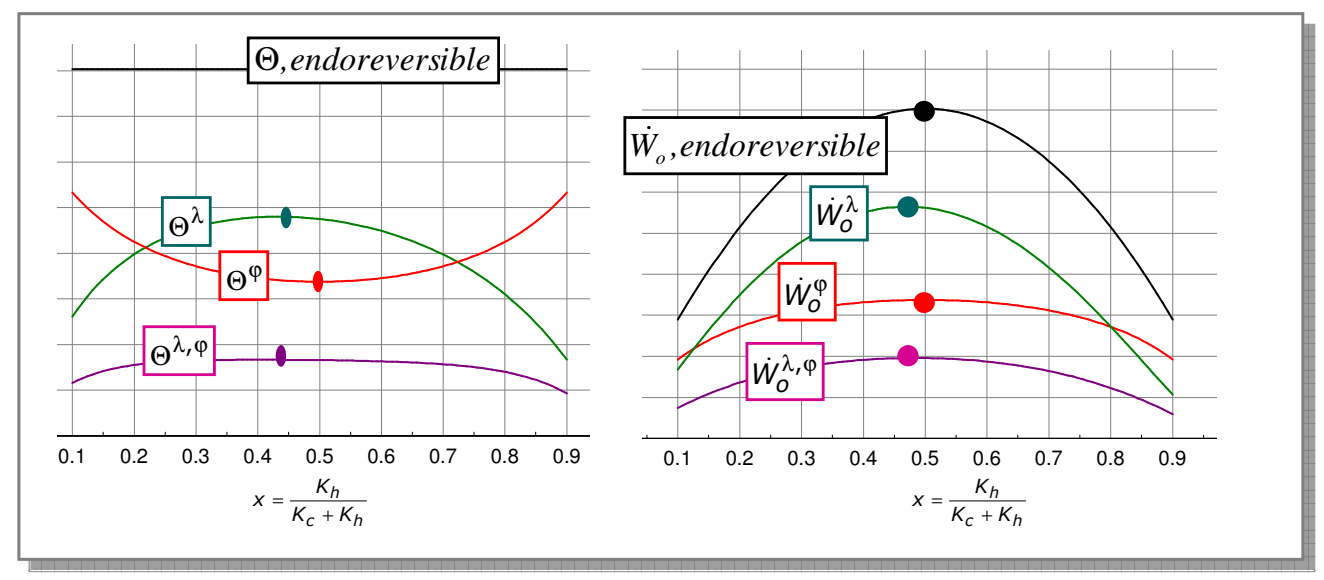

In the special case where the optimization constraint is the total conductance $K_{c}+K_{h}=c s t$, the parameter $\lambda$ and the conductance $K_{\lambda}^{\lambda}$ are constant. As a result, the conductance $K_{s c}^{\lambda}$ is maximum when the conductances $K_{c}$ and $K_{h}$ are equal. Finally, by taking into account the term $\Theta^{\lambda}$ (the calculations are heavy), it is shown that the output power $\dot{W}_{o}^{\lambda}$ is maximized for a $K_{h}$ slightly smaller than $K_{c}$. In the presence of internal dissipation and heat transfer, the result is intermediate, as shown in Figure 14.

\section{Conclusions and Perspectives}

The choice of selecting the entropy flow rate $\dot{S}$ involved in reversible energy conversion as the control variable of a heat engine has several advantages. We obtain, with a minimum of assumptions, a system of linear equations whose solution allows us to express all the variables (temperatures, thermal powers, mechanical power, etc.) as a function of the entropy flow rate $\dot{S}$. These analytical expressions associated with the analytical expression of the cutoff entropy flow rate enable the classical operating curves of the heat engine in $\left[\dot{W}, \eta_{1}\right]$ to be plotted.

By defining two dimensionless parameters $\lambda=\frac{2 K_{\lambda}}{K_{c}+K_{h}}$ and $\varphi=\frac{K_{s c} F^{\varphi}}{T_{s c}}$ where $K_{s c}=\frac{K_{c} K_{h}}{K_{c}+K_{h}}$ and $T_{s c}=\frac{K_{c} T_{c s}+K_{h} T_{h s}}{K_{c}+K_{h}}$, we quantified the intensity of internal dissipation and heat leakage within a heat engine, and we then established the analogy between an endoreversible heat engine and an irreversible heat engine by using the apparent temperatures $\left(T_{c s} \rightarrow T_{c s}^{\lambda, \varphi}, T_{h s} \rightarrow T_{h s}^{\lambda, \varphi}\right)$ and apparent conductances $\left(K_{h} \rightarrow K_{h}^{\lambda}, K_{c} \rightarrow K_{c}^{\lambda}, K_{s c} \rightarrow K_{s c}^{\lambda}\right)(c f$. Table 1$)$. We note that the apparent conductances are only affected by the internal heat leakage while the apparent temperatures are affected by both the internal dissipation and heat leakage. 
Table 1. Analogy between endoreversible heat engine and irreversible heat engine.

\begin{tabular}{ccc}
\hline Heat Engine Classification & Endoreversible Heat Engine & Irreversible Heat Engine \\
\hline Cutoff entropy flow rate: & $\dot{S}_{s c}=\frac{K_{c} K_{h}\left(T_{h s}-T_{c s}\right)}{K_{c} T_{c s}+K_{h} T_{h s}}$ & $\dot{S}_{s c}^{\lambda, \varphi}=\frac{K_{c}^{\lambda} K_{h}^{\lambda}\left(T_{h s}^{\lambda, \varphi}-T_{c s}^{\lambda, \varphi}\right)}{K_{c}^{\lambda} T_{c s}^{\lambda, \varphi}+K_{h}^{\lambda} T_{h s}^{\lambda, \varphi}}$ \\
Optimal entropy flow rate: & $\dot{S}_{o}=\frac{K_{c} K_{h}\left(\sqrt{T_{h s}}-\sqrt{T_{c s}}\right)}{K_{c} \sqrt{T_{c s}}+K_{h} \sqrt{T_{h s}}}$ & $\dot{S}_{o}^{\lambda, \varphi}=\frac{K_{c}^{\lambda} K_{h}^{\lambda}\left(\sqrt{T_{h s}^{\lambda, \varphi}}-\sqrt{T_{c s}^{\lambda, \varphi}}\right)}{K_{c}^{\lambda} \sqrt{T_{c s}^{\lambda, \varphi}}+K_{h}^{\lambda} \sqrt{T_{h s}^{\lambda, \varphi}}}$ \\
Maximum output power: & $\dot{W}_{o}=K_{s c}\left(\sqrt{T_{h s}}-\sqrt{T_{c s}}\right)^{2}$ & $\dot{W}_{o}^{\lambda, \varphi}=K_{s c}^{\lambda}\left(\sqrt{T_{h s}^{\lambda, \varphi}}-\sqrt{T_{c s}^{\lambda, \varphi}}\right)^{2}$ \\
\hline
\end{tabular}

The analytical expression of cutoff entropy flow rate is very important for engineers seeking to define the operating range of the machine, and the optimal entropy flow rate indicates the operating point where we obtain the maximum potential of the system specified by the expression of the maximum output power. In the application of a thermoelectric generator, we deduced easily the optimal electric current $I_{o}^{\lambda, \varphi}=\frac{\dot{S}_{o}^{\lambda, \varphi}}{\alpha}$ for which the output electric power is maximized [50].

However, these apparent temperatures should not be used to calculate the optimal energy conversion efficiency by analogy with the case of the endoreversible heat engine: $\eta_{o}^{\lambda, \varphi} \neq \eta_{C N C A}^{\lambda, \varphi}=1-\frac{\sqrt{T_{c s}^{\lambda, \varphi}}}{\sqrt{T_{h s}^{\lambda, \varphi}}}$.

In addition, the analytical expression of the maximum output power makes it possible to calculate without difficulty, at least numerically, the optimal allocation of conductances depending on the chosen optimization constraint. The application of our approach to a thermoelectric generator shows that the figure of merit ZT alone should not be used to characterize the performance of the system.

\section{References}

1. Dong, Y.; El-Bakkali, A.; Descombes, G.; Feidt, M.; Périlhon, C. Association of finite-time thermodynamics and a bond-graph approach for modeling an endoreversible heat engine. Entropy 2012, 14, 642-653.

2. Chen, L.; Wu, C.; Sun, F. Finite time thermodynamic optimization or entropy generation minimization of energy systems. J. Non-Equilib. Thermodyn. 1999, 24, 327-359.

3. Chen, L.; Wu, C.; Sun, F.; Chen, W. Optimal performance of an endoreversible Carnot heat pump. Energy Convers. Manag. 1997, 38, 1439-1443.

4. Chen, L.; Wu, C.; Sun, F.; Chen, W. General performance characteristics of finite speed Carnot refrigerator. Appl. Therm. Eng. 1996, 16, 299-303.

5. Wu, C.; Kiang, R.L. Finite-time thermodynamic analysis of a Carnot engine with internal irreversibility. Energy 1992, 17, 1173-1178.

6. Feidt, M. Optimal thermodynamics-New upperbounds. Entropy 2009, 11, 529-547.

7. Feidt, M.; Costea, M.; Petre, C.; Petrescu, S. Optimization of the direct Carnot cycle. Appl. Therm. Eng. 2007, 27, 829-839. 
8. Feidt, M. Reconsideration of criteria and modeling in order to optimize the efficiency of irreversible thermomechanical heat engines. Entropy 2010, 12, 2470-2484.

9. De Vos, A. Endoreversible thermoeconomics. Energy Convers. Manag. 1995, 36, 1-5.

10. Angulo-Brown, F. An ecological optimization criterion for finite-time heat engines. J. Appl. Phys. 1991, 69, 7465.

11. Curzon, F.L.; Ahlborn, B. Efficiency of a Carnot engine at maximum power conditions. Am. J. Phys. 1975, 53, 570-573.

12. Tyagi, S.K.; Chen, J.; Kaushik, S.C. Thermoeconomic optimization and parametric study of an irreversible Stirling heat pump cycle. Int. J. Thermal Sci. 2004, 43, 105-112.

13. Borel, L.; Favrat, D. de l'énergie à l'exergie. In Thermodynamique et énergétique (in French); Presses polytechniques et universitaires romandes: Lausanne, France, 2005; Volume 1, p. 814.

14. Bejan, A. Advanced Engineering Thermodynamics; Wiley: New York, NY, USA, 1988.

15. Chen, L.; Zhou, J.; Sun, F.; Wu, C. Ecological optimization for generalized irreversible Carnot engines. Appl. Energy 2004, 77, 327-338.

16. Chambadal, P. Les Centrales Nucléaires (in French); Armand Colin: Paris, France, 1957.

17. Novikov, I.I. The efficiency of atomic power station. J. Nucl. Energ. 1958, 7, 125-128.

18. Feidt, M. Thermodynamique et Optimisation Energétique des Systèmes et Procédés (in French); Lavoisier: Paris, France, 1987; ISBN: 2852063727.

19. Jou, D.; Casas-Vasquez, J.; Lebon, G. Extended Irreversible Thermodynamics, 3rd ed.; Springer Verlag: Berlin, Germany, 2001.

20. Andresen, B. Finite Time Thermodynamics; Physics Laboratory, University of Copenhagen: Copenhagen, Denmark, 1983.

21. Bejan, A. Entropy generation minimization: The new thermodynamics of finite size, and finite time processes. J. Appl. Phys. 1997, 79, 1191-1218.

22. Bejan, A.; Tsatsaronis, G.; Moran, M. Thermal Design and Optimization; J. Wiley and Sons Inc.: New York, NY, USA, 1996.

23. Goth, Y.; Feidt, M. Recherche sur les conditions optimales de fonctionnement des pompes à chaleur ou machines à froid associées à un cycle de Carnot endoreversible (in French). $C$. $R$. Acad. Sci. 1986, 303, 113-122.

24. Van den Broeck, C. Thermodynamic efficiency at maximum power. Phys. Rev. Lett. 2005, 95, 190602.

25. Jimenez de Cisneros, B.; Calvo Hernandez, A. Collective working regimes for coupled heat engines. Phys. Rev. Lett. 2007, 98, 130602.

26. Jimenez de Cisneros, B.; Calvo Hernandez, A. Coupled heat devices in linear irreversible thermodynamics. Phys. Rev. E 2008, 77, 041127.

27. Schmiedl, T.; Seifert, U. Efficiency at maximum power: An analytically solvable model for stochastic heat engines. Europhys. Lett. 2008, 81, 20003.

28. Apertet, Y.; Ouerdane, H.; Goupil, C.; Lecoeur, Ph. Irreversibilities and efficiency at maximum power of heat engines: The illustrative case of a thermoelectric generator. Phys. Rev. E 2012, 85, 031116.

29. Apertet, Y.; Ouerdane, H.; Goupil, C.; Lecoeur, Ph. Efficiency at maximum power of thermally coupled heat engines. Phys. Rev. E 2012, 85, 041144. 
30. Andresen, B.; Salamon, P.; Berry, R.S. Thermodynamics in finite time: Extremals for imperfect heat engines. J. Chem. Phys. 1977, 66, 1571-1577.

31. Andresen, B.; Salamon, P.; Berry, R.S.; Nitzan, A. Thermodynamics in finite time. I. The step-Carnot cycle. Phys. Rev. A 1977, 15, 2086-2093.

32. Andresen, B.; Salamon, P.; Berry, R.S. Thermodynamics in finite time. II. Potentials for finitetime processes. Phys. Rev. A 1977, 15, 2094-2102.

33. Andresen, B. Current trends in finite-time thermodynamics. Angew. Chem. Int. Ed. Engl. 2011, 50, 2690-2704.

34. Tu, Z.C. Recent advance on the efficiency at maximum power of heat engines. Chin. Phys. B 2012, 21, 020513.

35. Feidt, M. Thermodynamics of energy systems and processes: A review and perspectives. J. Appl. Fluid Mech. 2012, 5, 85-98.

36. Xuan, X.C. Optimum staging of multistage exo-reversible refrigeration systems. Cryogenics 2003, 43, 117-124.

37. Popescu, C.; Radcenco, V.; Costea, M.; Feidt, M. Optimisation thermodynamique en temps fini du moteur de Stirling endo- et exo-irréversible. Rev. Gin. Therm. 1996, 35, 656-661.

38. Vijay, P.; Samantaray, A.K.; Mukherjee, A. A bond graph model-based evaluation of a control scheme to improve the dynamic performance of a solid oxide fuel cell. Mechatronics 2009, 19, 489-502.

39. Boulon, L. Modélisation multi physique des éléments de stockage et de conversion d'énergie pour les véhicules électriques hybrides. Approche systémique pour la gestion d'énergie (in French). Ph.D. disertation, Université de Franche-Comté, France, July 2009.

40. Wu, C. Analysis of waste heat thermoelectric power generators. Appl. Therm. Eng. 1996, 16, 63-69.

41. Lenoir, B.; Michenaud, J.; Dauscher, A. Thermoélectricité: Des principes aux applications (in French). Technique de l'ingénieur 2010, Référence K730.

42. Durmayaza, A.; Sogub, O.S.; Sahin, B.; Yavuz, H. Optimization of thermal systems based on finite-time thermodynamics and thermoeconomics. Prog. Energy Combust. Sci. 2004, 30, 175-217.

43. Kodal, A.; Sahin, B.; Yilmaz, T. A comparative performance analysis of irreversible Carnot heat engines under maximum power density and maximum power conditions. Energy Convers. Manag. 2000, 41, 235-248.

44. Wang, T.; Zhang, Y.; Peng, Z.; Shu, G. A review of researches on thermal exhaust heat recovery with Rankine cycle. Renew. Sustain. Energy Rev. 2011, 15, 2862-2871.

45. Tchanche, B.F.; Lambrinos, Gr.; Frangoudakis, A.; Papadakis, G. Low-grade heat conversion into power using organic Rankine cycles-A review of various applications. Renew. Sustain. Energy Rev. 2011, 15, 3963-3979.

46. Goupil, C.; Seifert, W.; Zabrocki, K.; Müller, E.; Snyder, G.J. Thermodynamics of thermoelectric phenomena and applications. Entropy 2011, 13, 1481-1517.

47. Apertet, Y.; Ouerdane, H.; Glavatskaya, O.; Goupil, C.; Lecoeur, Ph. Optimal working conditions for thermoelectric generators with realistic thermal coupling. Europhys. Lett. 2012, 97, 28001.

48. Arias-Hernandez, L.A.; Barranco-Jiménez, M.A.; Angulo-Brown, F. Comparative analysis of two ecological type modes of performance for a simple energy converter. J. Energy Inst. 2009, 82, 223-227. 
49. Arias-Hernandez, L.A.; Angulo-Brown, F.; Paez-Hernandez, R.T. First-order irreversible thermodynamic approach to a simple energy converter. Phys. Rev. E 2008, 77, 011123.

50. Dong, Y.; El-Bakkali, A.; Descombes, G.; Feidt, M.; Périlhon, C. Assessment of the fuel saving enabled by the heat recovery of exhaust gases using a thermoelectric generator. In Proceedings of the Conference CAR2011 International Congress Automotive and Environment, Pitesti, Romania, 2-4 November 2011.

(C) 2012 by the authors; licensee MDPI, Basel, Switzerland. This article is an open access article distributed under the terms and conditions of the Creative Commons Attribution license (http://creativecommons.org/licenses/by/3.0/). 